\title{
El "gran cisma fenomenológico" y el "cisma \\ fenomenológico-existencial”. Sobre la continuidad en \\ la crítica contemporánea respecto del tránsito de Husserl \\ hacia el idealismo trascendental
}

\author{
George Heffernan \\ Merrimack College
}

Resumen: Se reconoce generalmente que hay dos cismas en la historia temprana del movimiento fenomenológico. El primero, el "gran cisma fenomenológico", comenzó entre los años 1905 y 1913 y, como muchos de sus jóvenes contemporáneos, por ejemplo Pfänder, Scheler, Reinach, Stein e Ingarden, rechazó la transformación husserliana de la fenomenología de las Investigaciones lógicas (1900/190I) -esto es, de la fenomenología como psicología descriptiva- a la fenomenología de Ideas I (1913) -esto es, la fenomenología como idealismo trascendental. El segundo, el "cisma fenomenológicoexistencial", tuvo lugar entre 1927 y 1933, desde que en Ser y tiempo (1927) la filosofía heideggeriana se apartó de la fenomenología transcendental husserliana de la conciencia y se encaminó hacia una analítica ontológica de la existencia humana como la vía hacia una interpretación de la pregunta por el sentido del Ser. Este ensayo no gira en torno ni al primero ni al segundo cisma per se, sino que trata de la relación entre los dos. Se sugiere que el primer cisma anticipa al segundo y que el segundo recapitula al primero, de manera que, aunque el primero pudiera haber ocurrido sin el segundo, el segundo no habría ocurrido, como fue el caso, sin el primero. Se indica que el segundo cisma yace temporalmente de manera más próxima al primero de lo que hasta la actualidad se ha apreciado. Con todo, el ensayo busca responder a la siguiente pregunta: icómo es que el "gran cisma fenomenológico" y el "cisma fenomenológico-existencial" se iluminan el uno al otro filosóficamente?

Palabras claves: Husserl - Heidegger - dos cismas fenomenológicos - fenomenología trascendental - "gran cisma fenomenológico" - "cisma fenomenológico-existencial".

Abstract: It is generally acknowledged that there were two schisms in the early history
of the phenomenological movement. The first, the Great Phenomenological Schism,
started between 1905 and 1913 , as many of his younger contemporaries, for example, 
Pfänder, Scheler, Reinach, Stein, and Ingarden, rejected Husserl's transformation of phenomenology from the descriptive psychology of the Logical Investigations (1900/I90I') into the transcendental idealism of Ideas I (1913). The second, the Phenomenological-Existential Schism, happened between 1927 and 1933, as it emerged that with Being and Time (1927) Heidegger's philosophy had moved away from Husserl's transcendental phenomenology of consciousness toward an ontological analytic of human existence as the way to an interpretation of the question of the meaning of Being. This paper is about neither the first schism per se nor the second schism per se but about the relationship between the two. It suggests that the first schism anticipated the second and the second recapitulated the first, so that, although the first could have occurred without the second, the second would not have happened as it did without the first. It also indicates that the second schism lies temporally much closer to the first schism than has been hitherto appreciated. Above all, the paper seeks an answer to this question: How do the Great Phenomenological Schism and the Phenomenological-Existential Schism illuminate one another philosophically?

Keywords: Husserl - Heidegger - two phenomenological schisms - transcendental phenomenology- "Great Phenomenological Schism” - "Phenomenological-Existential Schism"

\section{$\S 1$. Introducción: la pregunta por la relación entre los dos cismas}

Se reconoce generalmente que hubo dos cismas en la historia temprana del movimiento fenomenológico. El primero, el "gran cisma fenomenológico”, comenzó entre los años 1905 y 1913 y, como muchos de sus jóvenes contemporáneos, por ejemplo Alexander Pfänder (1870-194I), Max Scheler (18741928), Adolf Reinach (1883-1917), Edith Stein (1891-1942) y Roman Ingarden (1893-1970), rechazó la transformación husserliana de la fenomenología de las Investigaciones lógicas (1900/190I)' -esto es, de la fenomenología como psicología descriptiva- a la fenomenología de Ideas relativas a una fenomenología pura y una filosofía fenomenológica. Libro Primero: Introducción general a la fenomenología pura (1913) -esto es, la fenomenología como idealismo trascendental. El segundo, el “cisma fenomenológico-existencial”, tuvo lugar entre 1927 y 1933, desde que en Ser y tiempo (1927) la filosofía heideggeriana

I Cf. Hua X, pp. 237-268, específicamente pp. 238 y 253; Hua II, pp. 6-7, 9-12, 44-45, 48, $55-58,60,72$. 
se apartó de la fenomenología transcendental de la conciencia husserliana y se encaminó hacia una analítica ontológica de la existencia humana (Dasein) como el camino hacia a una interpretación de la pregunta por el sentido del Ser (Sein). Así mismo, se reconoce generalmente que el primer cisma es menos conocido que el segundo.

Este ensayo no gira en torno al primero ni tampoco al segundo cisma per se, sino que trata de la relación entre los dos. Indicaremos que no hay solamente una proyección innegable del primer cisma en el segundo, sino que hay también una abrumadora superposición entre ambos cismas y que, por tanto, la distinción cronológica entre ellos no es tan afinada como la datación estándar podría implicar. Sugerimos que el primer cisma anticipa al segundo y que el segundo recapitula al primero, de manera que, aunque el primero pudiera haber ocurrido sin el segundo, el segundo no habría ocurrido tal como sucedió sin el primero. Esta interpretación conduce a la inferencia de que el cisma entre la psicología descriptiva y la fenomenología transcendental es el horizonte apropiado sobre el cual comprender el cisma entre la fenomenología husserliana de la conciencia y la hermenéutica heideggeriana de la existencia. Desafortunadamente esta conexión ha sido mayormente abandonada.

La evidencia histórica en favor de esta interpretación de eventos es circunstancial pero convincente. De hecho, hay una considerable superposición temporal entre los dos cismas. Primero, el "gran cisma fenomenológico" continuó hasta bien entrada la época del "cisma fenomenológico-existencial". Asimismo, el primer cisma no surgió abrupta y súbitamente con Ideas I, pues Husserl venía practicando el método de la reducción fenomenológica en los manuscritos de Seefelder sobre el tema de la "individuación" desde los años 1905-1909, método que aclaró en La idea de fenomenología de $1907^{2}$. Adicionalmente, el segundo cisma ya se había iniciado durante la época del primero, pues este último estaba más bien iniciándose que llegando a su fin en 1913. Finalmente, el plantear una dicotomía entre los dos desarrollos oscurece la continuidad entre los mismos. Solo se necesita consultar la evidencia en lo concerniente al rompimiento entre Husserl y Heidegger. Esta evidencia se encuentra tanto en los trabajos publicados como no publicados de ambos, en su correspondencia 
y en las interpretaciones de tal evidencia por los estudiosos. Toda la evidencia indica que su rompimiento debe comprenderse como un proceso y como un evento. Aquí no intentamos añadir ninguna nueva evidencia, sino más bien comprender la evidencia ya existente de una forma diferente.

Sin embargo, este ensayo se enfoca principalmente en la evidencia filosófica. Ciertamente, existe una considerable coincidencia entre los asuntos que separaron a Husserl de los primeros fenomenólogos y aquellos que lo separaron de Heidegger. Aunque no es agotada por el debate entre realismo e idealismo, una breve lista de estas cuestiones incluye: la crítica de la naturaleza de la reflexión y del método de la reducción, el argumento a favor de la existencia del ego trascendental, el problema de la existencia del mundo externo, la justificación de la tesis de la intencionalidad y, sobre todo, la relación entre la fenomenología descriptiva y el idealismo trascendental. Estas coincidencias temáticas indican que, así como el "gran cisma fenomenológico" no trata únicamente sobre las diferencias filosóficas entre Husserl y Pfänder, Scheler, Reinach, Stein, Ingarden, entre otros fenomenólogos, sino también sobreaquellas existentes entre Husserl y Heidegger; así también el "cisma fenomenológicoexistencial" no trata solamente sobre las diferencias existentes entre Husserl y Heidegger, sino también sobre aquellas existentes entre Husserl y Pfänder, Scheler, Reinach, Stein, Ingarden, entre otros fenomenólogos. Muchos filósofos dentro del movimiento fenomenológico quedaron profundamente perplejos ante la primacía percibida de la teoría sobre la práctica, de la reflexión sobre la acción, de la lógica sobre la ética, de la esencia sobre la existencia, de la eternidad sobre la historia, de la ciencia sobre la vida, de los objetos sobre las cosas o, en una palabra, de la Bewusstsein sobre el Dasein. Dicho sin rodeos, estaban preocupados de que el desenvolvimiento metódico de la reducción trascendental y el enfoque riguroso centrado en "pensamientos en tanto pensamientos" (noemata) no condujeran hacia "las cosas en sí mismas" (die Sachen selbst), sino que los alejara de estas, lo cual diera como resultado una pérdida de acceso al Ser (Sein).

Con respecto a la historia humana del movimiento fenomenológico, una conexión fatídica entre el "gran cisma fenomenológico" y el "cisma fenomenológicoexistencial" yace en el hecho de que Husserl colocó primero sus esperanzas sobre el futuro de la fenomenología en Pfänder, quien fuera una figura clave del primer cisma. Con el pasar del tiempo, sin embargo, Husserl llegó a 
considerarlo como sumergido en el "ontologismo" y el "realismo" porque rechazaba la transformación de la filosofía husserliana ${ }^{3}$. Solo entonces Husserl transfirió sus esperanzas -aunque, nuevamente, solo de manera temporal-a Heidegger, quien fuera la figura dominante del segundo cisma. Irónicamente, Husserl luego tuvo que confesar a nadie más que a Pfänder su error en juzgar a Heidegger, tanto en cuanto a su filosofía como a su carácter ${ }^{4}$. Trágicamente, Reinach murió por su Vaterland en la Guerra Mundial el 16 de noviembre de 1917 y, así, no pudo suceder a Husserl en Friburgo 5 .

Sobre todo, este ensayo busca una respuesta a la siguiente pregunta: ¿cómo pueden el "gran cisma fenomenológico" yel "cisma fenomenológico-existencial" iluminarse el uno al otro filosóficamente?

\section{$\S 2$. Primera parte: un recuento "promedio" de la relación entre Husserl y Heidegger}

Precisamente en el sentido en el cual Heidegger emplea tales palabras en Ser y tiempo ${ }^{6}$, existe una comprensión promedio, diaria y vaga de la relación entre Husserl y Heidegger. "Uno" puede decir", a la manera de habladurías ${ }^{8}$, que se asemeja a algo así: Edmund Husserl (1859-1938) precede y prepara a Martin Heidegger (1889-1976). Husserl es el profesor de Heidegger y Heidegger es el estudiante de Husserl. Inicialmente son mentor y pupilo, luego maestro y aprendiz y, finalmente, colaboradores y colegas. Con el paso del tiempo desarrollan no solo una amistad, sino también una relación familiar, con Husserl como una suerte de figura paterna y Heidegger como una suerte de figura filial. Heidegger le dedica Ser y tiempo (1927) a Husserl y Husserl procura que Heidegger le suceda en su posición en el Departamento de Filosofía en la Universidad de Friburgo (1928). Pero luego algo, todos saben exactamente qué, sucede. Gradualmente, al principio de manera imperceptible y finalmente de manera inequívoca, su relación deviene tensa. Aunque sea

\footnotetext{
3 Cf. Hua Dok III, II, pp. I29-186 (“Correspondencia a Alexander Pfänder”).

4 Cf. Ibid., pp. 178-I86 (Carta a Alexander Pfänder, 6 de enero de 193I).

5 Cf. Ibid., pp. 187-208 (“Correspondencia con Adolf Reinach”).

6 Cf. Heidegger, Martin, Sein und Zeit, § 2; en adelante, SZ.

$7 \quad$ Cf. ibid., § 27.

8 Cf. ibid., § 35.
} 
difícil identificar algún evento en particular como aquel que, eventualmente, es el que ocasiona la ruptura final, su alejamiento como proceso gradual puede ser fechado entre los años 1927 y 1933. En cualquier caso, no puede haber duda de que un punto de inflexión es el intento fallido de Husserl y Heidegger de componer conjuntamente un artículo sobre fenomenología para la Enciclopedia británica (1927-1928). Tan pronto como Heidegger llega a Friburgo como su sucesor, rompe todo contacto con Husserl (1928). Entre el 6 y el 7 de abril de 1933, Husserl, un judío, es "destituido" de su posición en la universidad junto con todos los funcionarios públicos "no arios", como parte de una legislación discriminatoria del Partido Nacional Socialista, mientras que Heidegger se convierte en el rector de la Universidad de Friburgo el 21 de abril de 1933 y se une al NSDAP el I de mayo de 1933. El 27 de mayo de 1933, Heidegger imparte la brillantemente ambigua lección inaugural de su rectorado, "La autoafirmación de la universidad alemana", la cual condujo a un contemporáneo a observar que al final del discurso "uno no sabía si uno debía leer a los pre-socráticos o marchar junto a la Sección de Asalto (SA)"9. Los rumores proliferan sobre la relación entre Husserl y Heidegger, el más famoso de los cuales, aunque falso, puede estar expresado en el comentario que Hannah Arendt le hace a Karl Jaspers en 1946 respecto de que Heidegger era un "asesino en potencia" dado que le prohibió a Husserl el acceso a la Facultad de Filosofía de la Universidad de Friburgo ${ }^{10}$. Cuando Husserl muere en 1938, Heidegger se declara enfermo y no atiende a su funeral. Ello, entonces, constituiría la comprensión promedio, diaria y vaga de la relación entre estos dos pensadores. Por cierto, ella es muy superficial y debería, por tanto, ser sometida a un escrutinio escéptico.

\section{§ 3. Segunda parte: un análisis del recuento de Heidegger sobre su ruptura con Husserl}

La evidencia de la ruptura definitiva entre Husserl y Heidegger en los años 1927-1933 es convincente. Sin embargo, la evidencia de la ruptura temprana entre Husserl y Heidegger en los años previos a 1927 es también persuasiva. Es circunstancial, pero ello no le resta contundencia-al contrario. La clave para 
comprender la relación entre Husserl y Heidegger es cambiar de paradigma: en lugar de ver la publicación de Ser y tiempo como un mero terminus a quo, se trata también de verla como el terminus ad quem de su ruptura. Otra manera de exponer este punto es sostener que la publicación de Ideas I tuvo tanto que ver con la ruptura entre Husserl y Heidegger como también la aparición de Ser y tiempo. Sin embargo, también se podría decir que hubo dos fases en el proceso de su ruptura -entre 1913-1927 y entre 1927-1933- y que, hasta ahora, la atención se ha centrado demasiado en el segundo periodo y muy poco en el primero. Un juicio equilibrado debería atribuir un peso igualitario a ambos períodos.

Entendido debidamente, el escrito de Heidegger "Mi camino hacia la fenomenología" (1963/1969) es la llave para comprender su relación y ruptura con Husserl. Los problemas evidentes con el recuento de Heidegger son, por supuesto, que fue escrito mucho después de acontecidos los eventos que narra, que se trata solo del relato de Heidegger y no [incluye] también el de Husserl y que, por varias razones -tanto personales como profesionales-, Heidegger tiene interés en explicar la ruptura con su ex promotor de la mejor manera posible. En efecto, el ensayo proviene aproximadamente de la época de la famosa "Entrevista Spiegel" de Heidegger, cuyos aspectos revisionista e interesado fueron reconocidos hace tiempo". Además, el ensayo no es tanto un ejercicio filosófico sino más bien un tributo profesional a Hermann Niemeyer, el editor de obras fundacionales de fenomenología -como las Investigaciones lógicas de Husserl y el propio Ser y tiempo ${ }^{12}$ de Heidegger. Se trata de una breve y selectiva historia de la fenomenología contada desde la perspectiva de la casa de Niemeyer. Por ello, uno debería permanecer, prima facie, escéptico ante la exactitud del recuento de Heidegger en "Mi camino hacia la fenomenología”. Investigaciones independientes muestran, sin embargo, que otra evidencia proveniente de otras fuentes corrobora fuertemente el recuento

II Cf. Heidegger, Martin, “'Only a God Can Save Us': The Spiegel Interview (1966)”, en: Sheehan, Thomas (ed.), Heidegger: The Man and the Thinker, pp. 45-67. La versión original, del 23 de setiembre de 1966, fue publicada en Der Spiegel, 31 de mayo de 1976, pp. 193-219, bajo el título “Nurnoch ein Gott kann uns retten”. Cf. también Hachmeister, Lutz, Heideggers Testament: Der Philosoph, der "Spiegel” und die SS, 2da. edición, Berlín: Propyläen/Ullstein, 20I4, pp. 7-60 y 283-310.

12 Cf. Heidegger, Martin, “Mein Weg in die Phänomenologie” (1963/1969), en: GA I4, p. 92, nota editorial. En adelante, me referiré a este texto abreviando el título a "Mein Weg". Todas las traducciones de este artículo están hechas por mí, excepto cuando se señala lo contrario. 
de Heidegger, el cual es, entonces, consistente con aquella otra evidencia. Lo dicho se mantiene también para otras declaraciones de Heidegger en lo que concierne a su relación y ruptura con Husserl. El enfoque aquí está en la evidencia de "Mi camino hacia la fenomenología", que es relevante para la pregunta por la relación entre el "gran cisma fenomenológico" y el "cisma fenomenológico-existencial”.

Heidegger comienza "Mi camino hacia la fenomenología" haciendo un recuento de los pasos familiares en el recorrido de su desarrollo filosófico más temprano. Heidegger cuenta que, desde 1907, cuando él aún se encontraba en el Gymnasium, la disertación de Brentano Sobre el múltiple significado del ser según Aristóteles (1862) fue el "bastón y la vara" en sus "torpes intentos iniciales de adentrarse en la filosofía"'3. En 1908, su último año en el Gymnasium, Heidegger también leyó Sobre el ser: esbozo de ontología (1896) de Braig, el cual presentaba largos pasajes de Aristóteles, Santo Tomás de Aquino y Suárez, así como también "la etimología de palabras referidas a los conceptos ontológicos básicos”|4. En el invierno de 1909-1910, Heidegger comenzó sus estudios académicos en la Facultad de Teología de la Universidad de Friburgo y, habiéndose hecho tiempo para el estudio de la filosofía, así como habiendo también aprendido "de los diarios filosóficos" que la "manera de pensar" de Husserl estaba "determinada" por la de Brentano, tenía dos volúmenes de la primera edición de las Investigaciones lógicas de Husserl (1900/190I) sobre su escritorio desde un comienzo". Desde el inicio de "Mi camino hacia la fenomenología", Heidegger deja en claro que, desde sus "torpes intentos iniciales de adentrarse en la filosofía", su pregunta no trataba sobre el ente (Seiendes) sino sobre el ser (Sein): "Si ente [das Seiende] se dice de múltiples maneras, entonces ¿cuál es el significado básico anterior? ¿Qué es el ser [Sein]?”|6 De esta manera, la pregunta de Heidegger no era aquella de Husserl, la cual recaía sobre la Bewusstsein.

I3 Heidegger, Martin, ibid., p. 8I. Cf. Brentano, Franz, Von der mannigfachen Bedeutung des Seienden nach Aristoteles (1862).

14 Heidegger, Martín, “Mein Weg”, pp. 8I-82. Cf. Braig, Carl, Vom Sein: Abriß der Ontologie (I896).

I5 Cf. Heidegger, Martin, “Mein Weg”, p. 8I. Aparentemente hubo poca demanda por dicho trabajo en la Facultad de Teología. 
Consiguientemente, Heidegger cuenta que su actitud hacia la filosofía de Husserl fue ambivalente desde el inicio. De un lado, esperaba que las Investigaciones lógicas de Husserl fueran una "ayuda decisiva" en lo concerniente a las preguntas estimuladas por el libro de Brentano ${ }^{17}$. De otro lado, su esfuerzo por encontrar una respuesta a su propia interrogante sobre el ser con la ayuda del trabajo de Husserl fue "en vano, pues como aprendió solo de manera tardía, él no estaba buscando de la manera correcta"18. En el ínterin, no obstante, Heidegger permaneció tan fascinado por las Investigaciones lógicas de Husserl que las "leía repetidamente durante los siguientes años", pero "sin ganar mayor comprensión respecto de lo que le fascinaba"19. Del trabajo de Husserl emanaba "magia”, pero la comprensión de Heidegger sobre la fenomenología era "limitada y vacilante" ${ }^{20}$. En I9II, luego de pasar cuatro semestres en la universidad, Heidegger abandonó sus estudios teológicos y, en 1912, comenzó sus estudios filosóficos bajo la dirección de Heinrich Rickert (I863-1936), un importante neo-kantiano conocido por su trabajo en la teoría del conocimiento ${ }^{21}$.

De acuerdo con Heidegger, fue este su giro hacia la filosofía transcendental del conocimiento de Rickert, y especialmente a la de su pupilo Emil Lask (1875-1915), cuyos trabajos exhibían la influencia de las Investigaciones lógicas de Husser ${ }^{22}$, lo cual le "forzó nuevamente a trabajar en la obra de Husserl" 23 . Aun cuando no lo menciona en "Mi camino hacia la fenomenología”, Heidegger presentó en 1913 su tesis doctoral, "Sobre la doctrina del juicio en el psicologismo"24, con Artur Schneider (1876-1945) como director y con Rickert como segundo lector. En ella, reconocía la importancia de la contribución de Husserl en la batalla contra el psicologismo lógico, que era la posición dominante en el pensamiento alemán de aquel entonces y que sostenía que la psicología -no

17 Cf. ibid., p. 82.

18 lbid.

19 Ibid.

20 Ibid.

21 Cf. ibid., pp. 82-83. Cf. también Rickert, Heinrich, Der Gegenstand der Erkenntnis: Einführung in die Transzendentalphilosophie (I892).

22 Cf. Heidegger, Martin, “Mein Weg”, p. 83. Cf. también Lask, Emil, Die Logik der Philosophie und die Kategorienlehre: Eine Studie über den Herrschaftsbereich der logischen Form (I9II) y Die Lehre vom Urteil (1912).

23 Heidegger, Martin, “Mein Weg”, p. 83.

24 Cf. Heidegger, Martin, "Die Lehre vom Urteil im Psychologismus: Ein kritisch-positiver Beitrag zur Logik (1913/1914)”, en: GA I (1978), pp. I-I29. 
la filosofía- es la ciencia apropiada para abordar no solo los actos y los objetos del pensamiento, sino también los juicios lógicos mediante los cuales se expresan $^{25}$. Y, sin embargo, "el intento reiterado" de trabajar las Investigaciones lógicas de Husserl también permaneció "insatisfactorio" porque Heidegger no podía superar "la principal dificultad de cómo debía llevarse a cabo la manera de pensar que se llama a sí misma 'fenomenología"”26. La preocupación principal, dice Heidegger, resultaba de la "ambigüedad" en el trabajo de Husserl, la cual resaltaba "a primera vista" ${ }^{27}$. El punto decisivo del problema era-0, más precisamente, es- que el primer volumen de las Investigaciones lógicas refuta al psicologismo en lógica al demostrarse que las doctrinas del pensamiento y del conocimiento no pueden estar basadas en la psicología, mientras que el segundo volumen, Investigaciones sobre fenomenología y teoría del conocimiento, contiene las descripciones de los actos de la conciencia que son esenciales a la estructura del conocimiento ${ }^{28}$. Para Heidegger, entonces, el segundo volumen, que era tres veces más largo que el primero, parecía, después de todo, representar a la psicología y Husserl, con sus descripciones fenomenológicas de los fenómenos de la conciencia, parecía haber "recaído" en el psicologismo que acababa de refutar en su primer volumen ${ }^{29}$. Esto parecía sostenerse principalmente para el análisis de las experiencias intencionales o actos de la conciencia de la Quinta Investigación ${ }^{30}$. No obstante, Heidegger preguntaba, si tal "grave error" podía no atribuírsele al trabajo de Husserl, entonces iqué se supondría que representan las descripciones fenomenológicas de los actos de la conciencia?' ¿Qué se supone que es lo especial de la fenomenología si no se trata ni de psicología ni de lógica? ${ }^{32}$ ¿Se supone que es una nueva disciplina en filosofía, una de rango y precedencia? ${ }^{33}$ Heidegger refiere que él "no podía desenmarañar estas preguntas", que él "permanecía sin saber qué hacer o a dónde ir" y que en ese tiempo él "no podía siquiera formular las preguntas

25 Cf. ibid., p. 5 ss.

26 Heidegger, Martin, “Mein Weg”, p. 83.

27 Ibid.

28 Cf. ibid.

29 Cf. ibid. Husserl fue agudamente consciente de esta crítica, aunque no necesariamente como una lanzada por Heidegger. Cf., v. gr., Husserl, Edmund, "Entwurf einer 'Vorrede' zu den Logischen Untersuchungen", en: Hua XX/I, p. 279. A Husserl se le acusó de un "Rückfallen den Psychologismus".

30 Cf. Heidegger, Martin, “Mein Weg”, p. 83.

31 Cf. ibid.

32 Cf. ibid.

24233 Cf. ibid., pp. 83-84. 
con la claridad mediante la cual [estas] son expresadas" en "Mi camino hacia la fenomenología" ${ }^{34}$. Heidegger es cuidadoso en distinguir entre "ahora" y "en aquel entonces" en su recuento, de modo que no proyectase intuiciones poseídas en el presente hacia el pasado.

En consecuencia, ya en los años 1909 y 1913 surge un primer nivel de ambivalencia por parte de Heidegger respecto del trabajo de Husserl. En efecto, la actitud inicial de Heidegger de vacilación e incertidumbre hacia la fenomenología de Husserl es generada por lo que él percibía como la "ambigüedad" en la relación entre el primer y el segundo volumen de las Investigaciones lógicas. A ello pronto se le suma un segundo nivel de ambigüedad por parte de Husserl, el cual evoca un segundo nivel de ambivalencia para Heidegger.

Al seguir su historia en "Mi camino hacia la fenomenología", Heidegger inicialmente cuenta que "el año 1913 trajo una respuesta" a sus preguntas ${ }^{35}$. Sin embargo, luego él procede a revisar su declaración, a tal punto que sus declaraciones subsiguientes indican que la publicación de Ideas relativas a una fenomenología pura y una filosofía fenomenológica. Primer libro: Introducción general a la fenomenología pura de Husserl ese año introdujo un nivel de ambigüedad enteramente nuevo, que a su vez condujo a una mayor ambivalencia de su parte. Heidegger describe así a la "fenomenología pura" de Husserl: "La fenomenología pura" es la "ciencia fundamental" de la filosofía que es forjada por tal fenomenología. "Puro" significa "fenomenología trascendental". Y, sin embargo, la "subjetividad" del sujeto cognoscente, actuante y valorante está puesta como "trascendental". Ambos términos, "subjetividad" y "transcendental", muestran que la "fenomenología" consciente y decididamente se dirigió hacia la tradición de la filosofía moderna, pero de forma tal que "la subjetividad trascendental" alcanza una determinación más original y universal a través de la fenomenología. La fenomenología conserva las "experiencias de la conciencia" como su campo temático, pero ahora en la investigación sistemáticamente planeada y asegurada de la estructura de los actos de la experiencia en conjunto con la investigación de los objetos que son experimentados en tales actos con respecto a su objetividad ${ }^{36}$. 
Es de notar que Heidegger habla aquí de un "giro" (el verbo es "einschwenken" o literalmente un "oscilar en") de la fenomenología hacia la filosofía trascendental moderna. Así, plantea la cuestión de si este nuevo aproximamiento por parte de Husserl proveía de respuestas a las antiguas interrogantes que Heidegger veía planteadas por las Investigaciones lógicas. Dado que queda claro del resto del recuento de "Mi camino hacia la fenomenología" que Heidegger no responde a esta pregunta de manera afirmativa, debe entenderse que el movimiento husserliano de la fenomenología descriptiva de las Investigaciones lógicas hacia la fenomenología transcendental de Ideas I solo sirvió, según Heidegger, para crear otra ambigüedad de segundo nivel, la cual llevaría a otra ronda de ambivalencia de su parte. Su indecisión y vacilación, sin embargo, asumen gradualmente un carácter escéptico y de rechazo.

Que esta es, de hecho, la reacción de Heidegger, queda claro a partir del hecho de que, tal y como prosigue su relato, se niega a afirmar que las Ideas I de Husserl representaron un progreso en el proyecto fenomenológico de alcanzar "las cosas mismas" 37 ; más bien, testifica que tanto él como otros fenomenólogos permanecieron más interesados en las Investigaciones lógicas que en Ideas $I$. Esto sucedió incluso mientras, de acuerdo a Heidegger, Husserl introducía aún una tercera fuente de ambigüedad. A saber, en el intento de asignarle a las Investigaciones lógicas "un lugar sistemático en el proyecto universal de una filosofía fenomenológica" -que, como Heidegger sostiene, "han permanecido, por así decirlo, filosóficamente neutrales" con respecto al giro trascendental-Husserl publicó el trabajo en una segunda versión revisada y editada en $1913^{38}$. Sin embargo, esta acción también introdujo otra complicación dentro de la complicación mencionada, ya que solo los Prolegómenos y las Investigaciones I-V fueron publicadas en la segunda edición de tal año, aun cuando las Investigaciones I-V fueron sometidas a varios niveles de revisión que oscilan entre aquellos que dejaron subsistir "oscuridades", "errores" y "defectos" (I), hasta aquellos que lograron "una revisión muy completa" (III) y "revisiones de largo alcance" $(\mathrm{V})^{39}$. A saber, en el "Prefacio" a la segunda edición de la obra, al revelar su mirada esperanzadora en torno a la relación entre las

37 No afirmar, por supuesto, no es lógicamente equivalente a negar. Sin embargo, dado lo que debió haber sido la clara expectativa de Husserl -la afirmación por parte de Heidegger-, no afirmar y negar se acercan para todo propósito práctico. 
Investigaciones lógicas y el nuevo trabajo, Husserl concedió "la imposibilidad de elevar íntegramente el trabajo anterior al nivel de Ideas l", estableció sus "máximas de revisión" y afirmó que "en la última investigación", esto es, en la Quinta Investigación, “el nivel de ldeas [había sido] esencialmente alcanzado”40. Por otro lado, en el caso de la Sexta Investigación, "la más importante en lo que respecta a la fenomenología" (como anotan tanto Husserl como Heidegger), Husserl no quería hacer ninguna concesión en el sentido de sus máximas, de modo que retuvo su forma revisada hasta 1921 para poder introducirle una serie de nuevos capítulos ${ }^{41}$.

Incluso antes de examinar el tercer nivel de ambivalencia de su parte, debería quedar claro que Heidegger objeta, por decir lo menos, el desarrollo de la fenomenología husserliana de las Investigaciones lógicas hasta Ideas ${ }^{42}$. En lugar de reconocer la validez de "la obvia idea que a menudo circulaba en aquel tiempo", a saber, que en el año 1913 "una nueva dirección había tenido lugar en la filosofía europea"-la "fenomenología"-, Heidegger reta directamente la corrección de esta postura con una pregunta retórica: “QQuién habría querido negar la corrección de tal enunciado?"43 Y, no obstante, Heidegger hace e hizo exactamente esto, pues dice que "un error tal de cálculo histórico" falló en dar cuenta de "lo que había ocurrido con la 'fenomenología', esto es, ya desde las Investigaciones lógicas", a saber, que "las propias explicaciones programáticas y presentaciones metodológicas de Husserl más bien fortalecieron el malentendido que, a través de la 'fenomenología', se declaraba un comienzo de la filosofía el cual negaba todo pensamiento previo", y que este hecho "permaneció mudo y puede a duras penas ser correctamente expresado hoy en día", esto es, en $1963^{44}$. Poniendo fuera de duda que ya en 1913 él rechazó realizar "el giro trascendental" de Husserl, Heidegger establece simplemente: "Incluso luego de que Ideas relativas a una fenomenología pura fuera publicada, yo permanecía aún cautivado por el hechizo incesante que emanaba de las Investigaciones lógicas" 45 . "El hechizo", continua Heidegger, "trajo consigo nuevamente un

40 Cf. Hua XVIII, pp. 8-16, especialmente pp. I0-I5.

$4 I \quad C f$. Heidegger, Martin, “Mein Weg”, p. 84. Cf. Hua XIX/2, pp. 533-536.

42 Heidegger no asocia la anti-diltheyana "Filosofía como ciencia rigurosa" de Husserl con las Investigaciones lógicas, sino con Ideas. Cf. Heidegger, Martin, “Mein Weg”, pp. 84-85.

43 Ibid., p. 85.

44 Cf. ibid.

45 lbid. 
malestar cuya razón permanecía desconocida, aunque [le] hacía sospechar que venía de la falta de habilidad para alcanzar el acto del pensamiento filosófico denominado 'fenomenología' simplemente leyendo literatura filosófica" ${ }^{46}$. "La literatura filosófica” a ser leída ahora incluía, por supuesto, ia las Ideas relativas a una fenomenología pura y una filosofía fenomenológica de Husserl! Difícilmente puede haber una declaración más clara hecha por Heidegger respecto de que él fuera uno de los primeros "fenomenólogos" en resistirse a la transformación husserliana de la fenomenología descriptiva en una filosofía transcendental con su movimiento inevitable hacia un idealismo transcendental.

Sin embargo, Heidegger aún no ha terminado y continúa más bien relatando que "[su] perplejidad decayó lentamente y [su] confusión se disolvió laboriosamente solo desde que pudo encontrarse con Husserl personalmente en su centro de trabajo" 47 . Husserl, por supuesto, había llegado a Friburgo en 1916 como el sucesor de Rickert, quien había tomado el puesto de Windelband en Heidelberg. Sin embargo, una vez más, lo que Heidegger otorga con una declaración, lo quita con la siguiente, puesto que ahora aclara lo que dice haber aprendido de Husserl: "El dictado de Husserl tomó lugar a la manera de un entrenamiento paso-por-paso en la 'mirada' fenomenológica, la cual demandaba, al mismo tiempo, que uno abandonase el uso no comprobado del conocimiento filosófico, pero que, además, uno renunciase a introducir dentro de la conversación la autoridad de los grandes pensadores"48. La inferencia clara es o bien que Husserl es "un gran pensador" y que su "autoridad" no puede ser "introducida dentro de la conversación", o bien que no lo es, y entonces sí se puede. Sin embargo, mientras se le hacía más claro a Heidegger que "la creciente familiaridad con la mirada fenomenológica era fructífera para la interpretación de los escritos aristotélicos”, [tanto] menos era él capaz de "separarse [a símismo] de Aristóteles y de otros pensadores griegos"49. Aunque Heidegger agrega que él "no podía ver inmediatamente qué consecuencias decisivas [tendría] el renovado giro hacia Aristóteles" en su camino hacia la fenomenología, más tarde se le hizo claro que lo conduciría lejos de la nueva concepción de Husserl de una fenomenología trascendental de la conciencia

46 Ibid.

47 Ibid.

48 Ibid., p. 86.

24649 lbid. 
y sus contenidos, y [más bien] hacia una hermenéutica fenomenológica de la vida práctica ${ }^{50}$. Sin mencionar que se había convertido en el asistente de Husserl en 1919 (el 21 de enero), Heidegger narra que "él mismo, en el enseñar y el aprender en la cercanía a Husserl desde 1919", "practicó la mirada fenomenológica" y, al mismo tiempo, intentó llevar a cabo una "comprensión transformada de Aristóteles en un seminario" ${ }^{1}$. Con esta combinación de fenomenología y hermenéutica, su "interés se inclinó nuevamente hacia las Investigaciones lógicas, sobre todo, hacia la Sexta Investigación de la primera edición" 52 . Heidegger dice específicamente que la distinción entre una intuición sensorial y una categorial, trabajada en aquella investigación, "se reveló a sí misma [para él] dentro de su alcance para la determinación de la 'multiplicidad de significados de ser'”33.

En este punto, lo que hemos indicado como los tres niveles de ambigüedad por parte de Husserl y los correspondientes tres niveles de ambivalencia por parte de Heidegger caen todos en su lugar. El tercer nivel de ambigüedad por parte de Husserl emerge plenamente y genera un nivel de ambivalencia totalmente nuevo por parte de Heidegger. Al referirse a la relevancia de la distinción entre la intuición sensorialy la categorial en torno a una comprensión de la pregunta por la multiplicidad de significados de ser, Heidegger dice que, "por esta razón", "nosotros", "amigos y alumnos" de Husserl, "solicitamos al maestro una y otra vez" volver a publicar la Sexta Investigación, la cual había resultado difícil de conseguir ${ }^{54}$. Cediendo ante la presión, por decirlo de alguna manera, Husserl publicó en 192I la segunda edición de la Sexta Investigación "en la antigua forma" $y$ "solo mejorada esencialmente en algunas secciones" 55 . Sin embargo, Heidegger relata que Husserl hizo el deslinde de decir que él hizo esto solo para "los amigos del presente trabajo" y que al hacer esto estaba simultáneamente diciendo también que "él mismo ya no podría estar en buenos términos con las Investigaciones lógicas luego de la publicación de

50 La última conferencia impartida por Heidegger en Friburgo, en el semestre de verano de 1923, fue "Ontología: hermenéutica de la facticidad". Cf. GA 63.

5I Heidegger, Martin, “Mein Weg”, p. 86.

52 Ibid.

53 lbid.

54 Cf. ibid.

55 Heidegger erróneamente dice que el año fue 1922, pero ese fue el año en el que las Investigaciones lógicas fueron publicadas de manera completa y juntas por primera vez como parte de la segunda edición. 
Ideas" ${ }^{56}$. De acuerdo con Heidegger, entonces, "la pasión y el esfuerzo del pensamiento [de Husserl] en la nueva ubicación de su actividad académica [Friburgo], ahora más que nunca, había girado al desarrollo sistemático del programa presentado en Ideas" ${ }^{57}$. Heidegger y los otros pupilos, de otro lado, permanecieron fascinados con las Investigaciones lógicas y sus respectivas primeras ediciones, especialmente con la Sexta Investigación Lógica, esto es, sin los esfuerzos implementados por Husserl para elevarlas al nivel de Ideas.

Desde este punto en adelante, de acuerdo con Heidegger, Husserl lo “observó de manera benevolente, pero básicamente en desacuerdo, mientras trabajaba en las Investigaciones lógicas semanalmente en grupos especiales de trabajo con estudiantes mayores" 58 . De acuerdo con Heidegger, fue "sobre todo la preparación para este trabajo" lo que le "rindió frutos", y con ella aprendió "una cosa", primero como "un presentimiento" y luego como "una intuición fundada": "Lo que sucede para la fenomenología de los actos de conciencia como manifestación de los fenómenos mismos es pensado de manera incluso más original por Aristóteles y en toda la existencia y el pensamiento griegos como 'A $A \dot{\eta} \theta \varepsilon l \alpha$, como el des-ocultamiento de lo que-está presente, su ser revelado, su mostrarse a sí mismo" 59 . Heidegger declara que, en consecuencia, lo que nuevamente han encontrado las investigaciones fenomenológicas como "la actitud de soporte del pensamiento" resulta ser "el rasgo característico fundamental del pensamiento griego, si no ciertamente de la filosofía como tal"60. Evidentemente, Heidegger había descubierto "a un [nuevo] gran pensador", a saber, Aristóteles, e "introdujo" su "autoridad en la conversación"6l. A medio camino de convertirse en el asistente de Husserl (1919) y de publicar

56 Ibid. Cf. Hua XIX/2, p. 534.

57 Heidegger, Martin, “Mein Weg”, p. 86. Cf. ibid., pp. 86-87.

58 lbid., p. 87.

59 lbid.

60 lbid.

6I Heidegger dio esta primera conferencia sobre Aristóteles, "Interpretaciones fenomenológicas sobre Aristóteles. Introducción a la investigación fenomenológica”, en Friburgo el semestre de invierno 1921/1922 (cf. GA 61). Continuó con una conferencia con el mismo título en el semestre de verano en Friburgo en 1922 (cf. GA 62). En el otoño de 1922, Heidegger presentó sus interpretaciones sobre Aristóteles en Marburgo, donde estaba buscando una posición con la ayuda de Husserl (desde Friburgo), Paul Natorp (I854-1924) y Georg Misch (I878-1965). Habiendo obtenido la posición en Marburgo en 1923, Heidegger dio su primera conferencia sobre Aristóteles, "Conceptos básicos de la filosofía aristotélica", en el semestre de verano de 1924 (cf. GA 18). 
Ser y tiempo (1927), Heidegger le confió a Löwith que estaba considerando seriamente "si [él] debería retirar [su] Arist[óteles]" porque, si publicaba un libro que indicara su pensamiento en esta dirección, entonces "el anciano" (Husserl) "probablemente" notaría que le estaba "retorciendo el pescuezo" y "daría por terminado [el tema de] la sucesión" en Friburgo, solo para agregar que "[él] no podía controlarse [a sí mismo]", de modo que, al menos por un tiempo, seguiría yendo en la misma dirección ${ }^{62}$. La oblicua referencia atañe al hecho de que, en otoño del año 1922, Heidegger había delineado el bosquejo de un libro sobre Aristóteles, que debía aparecer en algún momento posterior a 1923 en el Anuario de Husserl, en el cual expondría su propia "hermenéutica de la facticidad" o "fenomenología de la vida"63. En un reporte de investigación sobre el trabajo, Heidegger ensalza a las Investigaciones lógicas de Husserl como un "descubrimiento" radical dentro de la investigación fenomenológica, pero critica la noción de que la fenomenología no deba ser nada más que un "análisis conceptual”64. También deja en claro que para él la fenomenología no es un análisis de la conciencia respecto de sus contenidos, sino una investigación de la "existencia humana" respecto de su "carácter de ser"65. Así definido por el giro de la vieja concentración husserliana en la Bewusstsein a un nuevo enfoque heideggeriano en el Dasein, el trabajo planeado sobre Aristóteles anticipaba "la fenomenología hermenéutica de la facticidad"66, de la vida fáctica de Ser y tiempo, por lo cual fue eventualmente suprimido.

Concluyendo la sección en "Mi camino hacia la fenomenología" sobre su relación con y ruptura respecto de Husserl, Heidegger cuenta que, mientras más veía la conexión entre la manifestación de los fenómenos en el sentido fenomenológico y el des-ocultamiento de lo que-está presente en el sentido griego, más presión sentía ante la pregunta: “iDe acuerdo al principio de la fenomenología, de dónde y cómo es determinado lo que debe ser experimentado en tanto 'la cosa en sí'? ¿[Acaso] es la conciencia y su objetividad, o [acaso] es el ser de los entes en su des-ocultamiento y ocultamiento?" ${ }^{67} \mathrm{Al}$

62 Cf. Heidegger, Martin, "Carta a Karl Löwith del 8 de mayo de 1923"; citado en: Thomä, Dieter (ed.), Heidegger Handbuch: Leben-Werk-Wirkung, p. 40.

63 Cf. GA 6I, p. 79 ss.

64 Cf. GA 62, pp. 364-365.

65 Cf. ibid., p. 348 ss.

66 Cf. ibid., p. 365.

67 Heidegger, Martin, “Mein Weg”, p. 87. 
responder la primera pregunta con una segunda pregunta, una retórica que contiene una clara disyunción, Heidegger indica que Husserly él estaban dando respuestas distintas a una misma pregunta. De esta manera, dice Heidegger, él fue traído al "camino de la pregunta por el ser", "iluminado por la actitud fenomenológica" $y$ "preocupado nuevamente pero de una forma distinta que antes" por las preguntas que habían emergido originalmente de la disertación de Brentano sobre el ser de acuerdo a Aristóteles ${ }^{68}$. Sin embargo, "la manera de preguntar", dice, devino "más extensa de lo sospechado" y requería "muchas paradas, desvíos y caminos errados"69. Al final, Heidegger nota que sus intentos [dados] en sus primeras lecciones en Friburgo y Marburgo para hacerse camino en lo relativo a "la forma de cuestionar" solamente muestran el camino indirectamente ${ }^{70}$.

Así, mientras Husserl se alejaba de las Investigaciones lógicas y [se encaminaba] hacia Ideas, dado que devenía crecientemente interesado en los análisis transcendentales de los contenidos, esto es, los actos y los objetos de la conciencia como tal, así también Heidegger se alejaba de Ideas de regreso a las Investigaciones lógicas, y en su primera edición, pues las consideraba más fructíferas y útiles en su búsqueda de una respuesta a la pregunta por el sentido del ser, inspirada por Aristóteles y los griegos, y recapitulada por Brentano ${ }^{71}$. Por lo tanto, desde el principio hasta el fin del involucramiento de Heidegger con la fenomenología y sus encuentros con Husserl, él estaba enfocado en investigar al Sein en términos del Dasein, mientras que Husserl permanecía fijado en investigar al Sein en términos de Bewusstsein. En este punto, no puede caber duda de que Heidegger veía la transformación de la fenomenología descriptiva en fenomenología transcendental e idealismo transcendental, como sucede en las Ideas relativas a una fenomenología pura y una filosofía fenomenológica, como un "camino equivocado" y que así [lo] pensaba en aquel tiempo.

68 Cf. ibid.

69 Ibid.

70 Cf. ibid. Cf. también el estudio de Matthias Jung sobre las conferencias de Heidegger en Friburgo y otros escritos entre 1919 y 1923, en: Thomä, Dieter (ed.), op. cit., pp. 13-21.

7I Cf. el estudio de Franco Volpi sobre el recurso de Heidegger a los griegos en la década de 1920 en: Thomä, Dieter (ed.), op. cit., pp. 25-35. Uno debería también examinar lo que dice Heidegger que aprendió de Braig sobre (I) la relevancia sobre la teología especulativa para la ontología y la metafísica y (2) la importancia del idealismo clásico germano (Schelling y Hegel) como una alternativa al escolasticismo. Cf. Heidegger, Martin, “Mein Weg”, pp. 8I-82. 
El recuento realizado por Heidegger sobre la génesis y la evolución de Ser y tiempo en "Mi camino hacia la fenomenología" es relativamente superficial y prácticamente vacío de contenido filosófico en comparación y en contraste con su descripción sobre su relación y ruptura respecto de la fenomenología Husserl, ya que su recuento se limita a hechos externos sobre la historia de la publicación del trabajo. Sin embargo, esta historia no es insignificante para comprender el lugar del camino filosófico de Heidegger en la historia de los dos cismas. A saber, Heidegger menciona que un día del semestre de invierno de 1925/1926, el decano de la Facultad de Filosofía de la Universidad de Marburgo entró a su oficina para preguntarle si tenía "un manuscrito apto" para su publicación ${ }^{72}$. La facultad había propuesto a Heidegger como professor ordinarius para suceder a Nicolai Hartmann (1882-1950), pero el 27 de enero de 1926, el Ministro de Cultura de Prusia en Berlín, Carl Heinrich Becker, había rechazado su propuesta "en razón de que [él] no había publicado nada en los últimos diez años"73. Heidegger narra que ya era el momento de "entregar [el] trabajo guardado durante largo tiempo"74. No obstante, solo después de un segundo rechazo por el Ministro (25 de noviembre de 1926) y de la publicación del trabajo en sí mismo (a fines de abril de 1927), Heidegger fue nombrado finalmente profesor ordinario en Marburgo (el 19 de octubre de 1927) ${ }^{75}$.

Lo absurdo de la situación puede fácilmente oscurecer el punto filosófico central que, en la medida en que no había publicado nada entre la aparición de La doctrina de las categorías y del significado de Duns Scoto, su Habilitationsschrift (dedicado a Rickert $)^{76}$ en 1916 y la consulta del decano en el invierno de 1925/1926, la publicación de Ser y tiempo en 1927 generó la impresión duradera -pero engañosa- de que el nuevo trabajo de Heidegger era el comienzo de su ruptura con Husserl, mientras que era, para todo propósito práctico, el fin de aquella ruptura, al menos filosóficamente hablando. Correspondientemente, en " $\mathrm{Mi}$ camino hacia la fenomenología”, Heidegger claramente ve la aparición de Ser y tiempo como anti-climática para el desarrollo de su relación con Husserl,

\footnotetext{
72 Cf. ibid., pp. 87-88.

73 lbid., p. 88.

74 lbid.

75 Cf. ibid. Él partió para Friburgo, claro está, en 1928.

76 El trabajo medieval en el cual el estudio de Heidegger estaba basado, De modis significandi, no es de Duns Scotus, como pensó Heidegger, sino de Thomas de Erfurt, como fue mostrado posteriormente por Martin Grabmann. Cf. Hua XVII, p. 54, nota al pie 2.
} 
y como su ocaso. Con respecto a la pregunta por la relación entre Husserl y Heidegger, de un lado, y a la pregunta por la relación entre el "gran cisma fenomenológico" y el "cisma fenomenológico-existencial”, del otro lado, es significativo que Heidegger -en sus observaciones detalladas en torno al método fenomenológico de investigación en Ser y tiempo ${ }^{77}$ - no haga mención a la reducción fenomenológica; elogia a las Investigaciones lógicas de Husserl "globalmente", pero a sus Ideas I "sólo de modo local"78, consistentemente busca al Sein no a través de la Bewusstsein, sino a través del Dasein.

En “Mi camino hacia la fenomenología”, entonces, Heidegger no está reinterpretando el pasado a la luz del futuro que ha devenido presente. Más bien, él está simplemente narrando lo él cree que ocurrió -so, wie es gewesen (Ranke). “Mi camino hacia la fenomenología” es la narración de la trayectoria del alejamiento Heidegger respecto del camino de Husserl en la fenomenología. Esta fuente, aunque compuesta en 1963 y retocada en 1969, es consistente con lo que se sabe del desarrollo de Heidegger entre su estudio de las Investigaciones lógicas de Husserl y su lectura de Ideas $I$. Las ambigüedades involucradas en cambios filosóficos de Husserl, (I) del primer al segundo volumen de las Investigaciones lógicas, (2) de las Investigaciones lógicas a Ideas relativas a una fenomenología pura y una filosofía fenomenológica l y (3) de la primera edición de las Investigaciones lógicas a la segunda edición de la obra, todos combinados convencen a Heidegger de que él prefería que la fenomenología no se transformara en [un] idealismo trascendental. Él anduvo constantemente intrigado por las preguntas que las Investigaciones lógicas planteaban, pero consistentemente no pensaba que Ideas I proporcionara las respuestas. Por el contrario, sugiere que el paso del trabajo temprano al posterior representa no un avance hacia, sino un retroceso que lo aleja de "las cosas mismas"79. Así, la ruptura temprana de Heidegger respecto de Husserl es parte del "gran cisma fenomenológico" y antecede por mucho al "cisma fenomenológico-existencial”. Y Heidegger así lo pensaba en aquel tiempo.

77 Cf. SZ, § 7. Esto no cambia en otras partes del trabajo.

78 Cf. ibid., pp. 38, 47, 77, 166, 218, 363.

25279 Cf. Pöggeler, Otto, Der Denkweg Martin Heideggers, pp. 67-80. 


\section{$\S$ 4. Tercera parte: evidencia corroborativa en torno a la precisión del recuento de Heidegger}

A primera vista, claro está, "Mi camino hacia la fenomenología" de Heidegger podría parecer como un intento ex post para trazar una mayor distancia ante post entre sí mismo y Husserl. Como se ha mostrado, sin embargo, el ensayo de Heidegger tiene una fuerte pretensión de presentar una explicación coherente y precisa sobre su distanciamiento gradual y, finalmente, sobre su ruptura definitiva. De hecho, la ruptura temprana entre Husserl y Heidegger está documentada no solo por la narrativa propia de Heidegger, sino también por una larga lista de fuentes contemporáneas, especialmente (por) su correspondencia, tanto entre ellos mismos como entre ellos y otros. Luego de revisar este conjunto de evidencia corroborativa, uno podría seguir preguntándose, en primer lugar, si Husserl y Heidegger estuvieron alguna vez genuinamente cerca, filosóficamente hablando.

Habría que seguir la evidencia. A inicios de 1917, apenas a un año de la llegada de Husserl a Friburgo como sucesor de Rickert en $1916^{80}$, Heidegger le dice a su esposa Elfride que no puede aceptar la fenomenología de Husserl como "irrevocable" porque, en términos de su "aproximación" y su "meta", "ella es [...] muy estrecha y [muy] insípida", mientras que "la vida es muy rica y muy grandiosa", de modo que alguien que es "solo un lógico" en búsqueda de "lo absoluto" no puede encontrar "el camino liberador" hacia "una configuración de las relatividades" ${ }^{\prime}$. En 1919, Heidegger escribe sobre la "conciencia histórica" y "la vida" -en el sentido de Dilthey- a la hija de Husserl, Elizabeth, diciendo que "si nuestra vida, al tomar forma, realmente vive su vida histórica", "todo depende del hecho de que ella misma sea", pero no en "la observación teórica de esta posibilidad" ni tampoco en "la reflexión sobre ella"82. En 1919, Heidegger también escribe a Elfride que, aunque "con muchos más horizontes

80 Husserl fue nombrado Ordinarius el 9 de febrero y se mudó a Friburgo el I de abril de 1916. Cf. Schuhmann, Karl (ed.), Husserl-Chronik: Denk- und Lebensweg Edmund Husserls, Hua Dok I, pp. 199-200. La primera correspondencia entre Husserl y Heidegger data de mayo de 1916 y su primer encuentro del domingo 23 de julio de 1916. Cf. Hua Dok III, IV, p. 127 (“Correspondencia entre Husserl y Heidegger”, pp. 127-16I).

8I Heidegger, Martin, "Carta a Elfride Heidegger del 27 de mayo de 1917", en: Heidegger, Martin. "Mein liebes Seelchen!" Briefe Martin Heideggers an seine Frau Elfride (1915-1970), p. 57. 82 Cf. Heidegger, Martin, "Brief Martin Heideggers an Elisabeth Husserl” (24 de abril de 1919), en: Aut- aut, 223-224 (1988), pp. 6-II. 
y problemas", él está "por encima y más allá de [Husserl]", él ha escogido la "cooperación" con él por consideraciones "personales" que son menos importantes que las "científicas" -y las "prácticas" ${ }^{3}$. A principios de 1920, además, Heidegger describe a Elfride cómo ellos se encuentran juntos "en camino hacia una genuina, simple y elemental aprehensión de la vida", pero cómo él está "separado" de Husserl por "mundos", con un "gran contraste" entre ellos y que, por ello, "solamente para preservarnos materialmente", él debe hallar una manera de llevarse bien con él "sin conflicto violento" ${ }^{4}$. Estas declaraciones, en combinación con el relato posterior de su camino hacia la fenomenología, indican que mucho antes de los años 1927-1931, y ciertamente mucho antes de los años 1933-1934, Heidegger era un crítico de la fenomenología de Husserl, y que su crítica filosófica se debía en gran medida a su rechazo al giro realizado por su antiguo mentor de una fenomenología entendida como psicología descriptiva en las Investigaciones lógicas (1900/190I, primera edición) a una fenomenología redefinida como idealismo trascendental en Ideas relativas a una fenomenología pura y una filosofía fenomenologías $(1913)^{85}$.

Sin embargo, Heidegger no expresa su antipatía hacia Husserl y su nueva fenomenología solo a su esposa. A principios de 1923, por ejemplo, Heidegger le cuenta a Löwith que ha "quemado y destruido" Ideas de Husserl en su seminario, así como también que, mirando hacia atrás a las Investigaciones lógicas, había llegado a "la convicción de que Husserl nunca fue un filósofo, ni siquiera por un segundo de su vida" ${ }^{86}$. Adicionalmente, para el verano de 1923, Heidegger escribe a Jaspers que Husserl "se ha desarmado completamente [...] si es que alguna vez estuvo 'en una sola pieza”, que Husserl "está hablando tantas trivialidades que uno debe tenerle lástima", que "vive de la misión del 'fundador de la fenomenología”, aunque "nadie sabe qué es eso", y que "está comenzando a sospechar que la gente ya no lo sigue" ${ }^{87}$. Durante

83 Cf. Heidegger, Martin, “Carta a Elfride Heidegger del 30 de agosto de 1919”, en: op.cit., pp. 95-96.

84 Cf. Heidegger, Martin, "Carta a Elfride Heidegger del 4 de enero de 1920”, en: ibid., pp. $103-104$.

85 Cf. nuevamente Heidegger, Martin, “Mein Weg”, pp. 8I-90 y Pöggeler, Otto, op. cit., pp. 67-80.

86 Heidegger, Martin, “Carta a Karl Löwith del 20 de febrero de 1923”, citado en: Thomä, Dieter (ed.), op. cit., p. 40.

87 Heidegger, Martin, “Carta a Karl Jaspers del 14 de julio de 1923”, en: Heidegger, Martin y Karl Jaspers, Briefwechsel 1920-1963, p. 42. 
el trabajo de Ser y tiempo en la primavera de 1926, Heidegger informa a Jaspers que Husserl "encuentra todo el asunto desconcertante y [que] 'ya no se acomoda' en la fenomenología usual", con lo cual Heidegger concluye que él está “de facto ya más alejado" de Husserl de lo que él mismo pensaba ${ }^{88}$. Finalmente, en el invierno de 1926, Heidegger escribe a Jaspers que, si Ser y tiempo está "escrito 'en contra de alguien', entonces [lo está] en contra de Husserl”, agregándole que Husserl "también lo notó inmediatamente” pero "desde el principio se apegó a lo positivo" ${ }^{9}$.

De hecho, Husserl había sentido, aunque vaga y oscuramente, gran parte de la alienación filosófica entre él mismo y Heidegger mucho antes de que aludiera a ello en sus escritos públicos y conferencias. Durante su intento fallido de co-autorar un artículo [titulado] "Fenomenología" para la Enciclopedia británica en $1927-1928^{90}$, por ejemplo, fue aparente que, mientras Husserl insiste en una separación aguda entre el ego puro trascendental y el ser humano natural, Heidegger rechaza esta aproximación egológica en tanto metafísica y aboga por la unidad del ego trascendental y la existencia fáctica que es un ser humano - una brecha demasiado amplia9'. Dicho sin rodeos: para Husserl, la Bewusstsein constituye al Sein, mientras que para Heidegger [el] Sein se revela [él mismo] al Dasein. Al término de 1927, en medio del fracaso en alcanzar un consenso en torno al artículo para la Enciclopedia británica, Husserl le informa a Ingarden que "lamentablemente [él] no había determinado la educación filosófica [de Heidegger]" y que "[Heidegger] aparentemente ya era extraño al estudiar los escritos [de Husserl]" ${ }^{92}$. En 1928, tan pronto como Heidegger llega a Friburgo como su sucesor, rompe todo contacto con Husser ${ }^{93}$. Finalmente, en el verano de 1929, luego de haber sido frecuentemente advertido

88 Cf. Heidegger, Martin, “Carta a Karl Jaspers del 24 de mayo de 1926”, en: ibid., p. 64.

89 Heidegger, Martin, "Carta a Karl Jaspers del 26 de diciembre de 1926", en: ibid., p. 7I.

90 Cf. Husserl, Edmund, “Der Encyclopaedia Britannica Artikel” (1927-1928), en: Hua IX, pp. 237-30I.

91 Cf. Husserl, Edmund, "Carta de Heidegger del 22 de octubre de 1927" y "Carta a Heidegger del 8 de diciembre de 1927”, en: Hua Dok III, IV, pp. I44-I48 y 148-I49 respectivamente, así como Hua IX, pp. 274-275.

92 Husserl, Edmund, “Carta a Roman Ingarden del 19 de noviembre de 1927”, en: Hua Dok III, III, p. 234.

93 Al menos según Husserl. Cf. Husserl, Edmund, "Carta a Dietrich Mahnke del 4/5 de mayo de 1933 (con Malvine Husserl)”, en: ibid., pp. 49I-502, específicamente p. 493. 
de que la fenomenología de Heidegger era diferente y hostil a la propia ${ }^{94}$ y ante las negativas desdeñosas de Heidegger respecto de tales rumores como "sinsentidos", Husserl estudiaba Ser y tiempo así como otros trabajos de su antiguo asistente para poder dar con "una posición sobria y final en torno a la filosofía heideggeriana" 95 , solo para llegar a "la inquietante conclusión de que [él] no tenía nada que ver con esta profundidad heideggeriana, con esta falta de cientificidad ingeniosa, y que la crítica abierta y oculta de Heidegger estaba basada en un craso malentendido, pues éste estaba involucrado en la elaboración de un sistema filosófico cuyo tipo [Husserl] siempre había considerado [que] [su propio] trabajo de una vida debía imposibilitar para siempre"96. Solo le quedaba a Husserl suspirar: "Todos los demás se percataron de esto hace ya tiempo -solo yo no lo hice"97.

En 1930, Husserl reafirma enfáticamente su giro transcendental en Ideas con un "Epílogo"98, en el cual también critica la "filosofía de la 'existencia”, pero sin mencionar a ninguno de los "filósofos de la existencia" por su nombre". En 1931, además, en una conferencia pública titulada "Fenomenología y antropología”, Husserl insiste en la importancia de la reducción transcendental como el punto de partida indispensable de una filosofía fenomenológica fundada metodológicamente, y esta vez demarca incisivamente su propia posición de las aproximaciones de Dilthey y Scheler, dando nombres ${ }^{100}$. Aunque no mencionó a Heidegger por su nombre en ninguno de los dos lugares, se pensó que

94 Y luego de escuchar la conferencia inaugural en Friburgo: “¿Qué es la metafísica?” (24 de julio de 1929).

95 Las anotaciones marginales de Husserl a Ser y tiempo y Kant y el problema de la metafísica de Heidegger han sido editadas por Roland Breeur ("Randbemerkungen Husserls zu Heideggers Sein und Zeit und Kant und das Problem der Metaphysik", en: Husserl Studies XI [1994], pp. 3-63). 96 Husserl, Edmund, "Carta a Alexander Pfänder del 6 de enero de 193I", en: Hua Dok III, II, pp. I80-184. Aparte de la carta de Husserl a Dietrich Mahnke del 4/5 de mayo de 1933 (en: Hua Dok III, III, pp. 49I-502), esta es la fuente más importante para ver cómo él concibió su ruptura con Heidegger.

97 Husserl, Edmund, “Carta a Alexander Pfänder del 6 de enero de 193I”, en: Hua Dok III, II, p. 184.

98 Cf. Husserl, Edmund, "Nachwort zu meinen Ideen zu einer reinen Phänomenologie und phänomenologischen Philosophie” (1930), en: Hua V, pp. 138-162, específicamente pp. I5I-I52, 154-155, 161.

99 En Neue Wege der Philosophie: Geist-Leben-Existenz. Eine Einführung in die Philosophie der Gegenwart (1929), Heinemann caracterizó la filosofía de Heidegger en SZ como “Existenzphilosophie”. 100 Cf. Husserl, Edmund, "Phänomenologie und Anthropologie" (193I), en: Hua XXVII, pp. 164-I8I, específicamente pp. 164 (Dilthey) y 180 (Scheler, Dilthey). Husserl presentó dicha conferencia en junio de 1931 en el Kantgesellschaft en Frankfurt, Berlín y Halle. 
Husserl también lo tenía en mente y, correcta o incorrectamente, Heidegger también pensó lo mismo ${ }^{101}$. Estos eventos también contribuyeron a una percepción pública [en torno a] que la ruptura entre Husserl y Heidegger fue el momento definitivo del "cisma fenomenológico-existencial", cuando de hecho fue un momento definitorio del "gran cisma fenomenológico". En general, se reconoce que Heidegger rechazó tanto a [la] "filosofía de la existencia” como [al] "existencialismo" como designaciones apropiadas para su filosofía del Ser ${ }^{102}$.

El giro político de Heidegger hacia el Nacional Socialismo tuvo naturalmente un severo impacto negativo en su relación personal con Husserl. Por supuesto, estos pensadores se habían crecientemente apartado durante los años de 1927-1931 ${ }^{103}$. En 1933-1934, sin embargo, no solo se presentaron diferencias filosóficas, sino también políticas, mientras Husserl presencia el emergente nacionalsocialismo de Heidegger y su creciente antisemitismo ${ }^{104}$. El 6 de abril de 1933, Husserl, con todos los "no-arios" funcionarios de Baden, fue destituido ("beurlaubt") de su posición en la universidad como medida del Reichskommissar regional ${ }^{105}$. Husserl llamó a esta medida y a la medida del 7 de abril de 1933 [a aplicarse] en todo el Reich, la "Ley para la Restauración del Servicio Civil Profesional” (Gesetz zur Wiederherstellung des Berufsbeamtentums), "el daño personal más grande de [su] vida"106. No obstante, el Ministro de Cultura de

I0I Cf. Heidegger, Martin, "Nur noch ein Gott kann uns retten”, específicamente p. 199. Cf. también Schuhmann, Karl, “Zu Heideggers Spiegel-Gespräch über Husserl” (1978), pp. 59I-612. Heidegger supo del contenido de dichas conferencias parcialmente por informes periodísticos. Cf. finalmente GA 97, p. 462, donde Heidegger dice que en conferencias públicas de “1930/3I”, Husserl "tomó una posición en contra [suya] y rechazó [su] trabajo como no-filosófico", y añade que Husserl hizo lo mismo en el "Epílogo a Ideas" de "I930/3I".

102 Cf. Heidegger, Martin, "Über den Humanismus" (1945), en GA 9; GA 27, p. 374; GA 32, p. 18; GA 49, § IIb; “Carta a Elisabeth Blochmann de 1947”, en: Heidegger, Martin y Elisabeth Blochmann, Briefwechsel 1918-1969, p. 93; “Carta de Karl Jaspers del 6 de febrero de 1949”, en: Heidegger, Martin y Karl Jaspers, op. cit., pp. 168-17I, 276-278. Cf. también Vetter, Helmuth, Grundriss Heidegger: Ein Handbuch zu Leben und Werk, pp. 265-266. La designación de la filosofía de Heidegger como "existencialismo" se halla, sobre todo, en el "Existentialismo es un humanismo" de Sartre, y como "filosofía de la existencia" ("Existenzphilosophie") se rastrea a las obras de Heinemann.

103 Cf. Sheehan, Thomas y Richard Palmer (eds.), Edmund Husserl: Psychological and Transcendental Phenomenology and the Confrontation with Heidegger (1927-1931), pp. I-32, así como Thomä, Dieter (ed.), op. cit., pp. 35-44.

104 Cf. Husserl, Edmund, "Carta a Dietrich Mahnke del 4/5 de mayo de 1933 (con Malvine Husserl)”, en: Hua Dok III, III, pp. 49I-502.

105 Cf. Hua Dok I, p. 428.

106 Cf. Husserl, Edmund, "Carta a Gustav Albrecht del I de julio de 1933", en: Hua Dok III, IX, p. 92: “(...) daß ich das neue Beamtengesetz und dann die Beurlaubung als größte Kränkung 
Baden cancela ("con reservas") la medida respecto de Husserl el 28 de abril; la cancelación fue confirmada el 20 de julio y, en su favor, el Rector Heidegger firmó la cancelación el 28 de julio ${ }^{107}$. Aun así, el 15 de enero de 1936, a Husserl se le extirpó terminal e irrevocablemente su permiso para dictar (venia legendi or Lehrbefugnis), a hacerse efectivo retroactivamente al 31 de diciembre de $1935^{108}$. Estos eventos y acontecimientos relacionados, acompañados por los episodios depresivos de Husserl y la carencia de solidaridad de Heidegger ${ }^{109}$, finalizaron lo que Husserl habría llamado agriamente "esta supuesta amistad filosófica entre almas" 110 . No cabe la menor duda de que Husserl vio una directa correlación entre el emergente compromiso con el partido Nacional Socialista y el anti-semitismo, de un lado, y el declive y colapso de su propia relación con Heidegger, de otro lado'"'. Al final, Heidegger, alegando [una] enfermedad, no asistió al funeral de Husserl el 29 de abril de 1938, un "error humano" del cual él se disculparía más adelante -en 1950-, con una nota y un bouquet de flores a la viuda de Husserl ${ }^{112}$. La división política entre Husserl y Heidegger convirtió la ruptura filosófica entre ellos en una ruptura absolutamente irreversible.

En el interés de un recuento balanceado, debería notarse que, en el clímax de sus buenas relaciones, Husserl no solamente aceptó Ser y tiempo en su Anuario de Filosofía e Investigación Fenomenológica, sino que "con alegría” leyó

meines Lebens empfunden habe".

107 Cf. Hua Dok I, pp. 429, 433. Cf. el malentendido temporal de estos acontecimientos por Arendt y Jaspers, en: Arendt, Hannah y Karl Jaspers, op. cit., pp. 79, 84, 99, 732.

108 Cf. Hua Dok I, p. 472.

109 Incluso la frecuentemente citada carta de Elfride Heidegger a Malvine Husserl del 29 de abril de 1933 es una débil excepción a la regla. También contiene la observación sin una demarcación temporal: "Wenn mein Mann in seiner Philosophie andere Wege gehen musste..." (Briefwechsel IV, pp. 160-16I). Sin embargo, no es cierto que Heidegger, como Rector, prohibiera a Husserl entrar a la Universidad de Friburgo. Cf. Heidegger, Martin, "Carta al editor de Der Spiegel" (22 de febrero de 1966), en: GA 16, p. 639. Cf. también GA 97, p. 462.

II0 Husserl, Edmund, "Carta a Dietrich Mahnke del 4/5 de mayo de 1933 (con Malvine Husserl)”, en: Hua Dok III, III, p. 493.

III Cf. ibid., otra vez: "[Der schönste Abschluß dieser vermeintlichen philosophischen Seelenfreundschaft war der (ganz theatralisch) am I. Mai öffentlich vollzogene Eintritten die Nationalsozialistische Partei. Vorangegangen ist der von ihm [Heidegger] vollzogene Abbruch des Verkehrs mit mir (und schon bald nach seiner Berufung) und in den letzten Jahren sein immer stärker zum Ausdruck kommender Antisemitismus -auch gegenüber seiner Gruppe begeisterer jüdischer Schüler unden der Fakultät.] Das zu überwinden, war ein schweres Stück".

II2 Cf. Heidegger, Martin, “Carta a Malvine Husserl del 6 de marzo de 1950", en: GA I6, p. 443. Cf. también Ott, Hugo, Martin Heidegger: Unterwegs zu seiner Biographie, pp. I67-I68. 
impresiones de prueba para su publicación (1926) ${ }^{113}$. Heidegger entusiastamente dedicó Ser y tiempo (1927) a Husserl ${ }^{114}$. Incluso mientras luchaban contra lo inevitable en otoño del927, Heidegger llamó a Husserl "querido amigo paternal”, y Husserl llamó a Heidegger "querido amigo"|l5. Al recomendarlo como "entre los maestros filosóficos más significativos de nuestro tiempo" 116 , Husserl se aseguró de que solamente Heidegger fuera enlistado como posible sucesor a su cátedra en el Departamento de Filosofía de la Universidad de Friburgo (1928) $)^{117}$. Incluso luego de que hubiera finalizado esencialmente su relación filosófica, ellos aún tenían ocasionalmente contacto social. Por ejemplo, Husserl invitó a Heidegger a su casa para un "té de filósofos" el 22 de junio de 1930 y por el cincuentenario de su propio doctorado el 23 de enero de 1933 (una semana antes de que Hitler fuera nombrado canciller), y Heidegger aceptó ambas invitaciones ${ }^{118}$. De modo que la historia a narrarse no es del todo negativa.

Aun así, es perfectamente comprensible cómo ya en 1946 Heidegger pudo escribir, en un ensayo que sería publicado por primera vez el año 2000 , que él "desde el inicio y siempre estuvo alejado de la posición filosófica de Husserl en el sentido de una filosofía trascendental de la conciencia"|l'. Todo el tiempo, dice Heidegger, ni había buscado encontrar "una nueva dirección dentro de la fenomenología" ni tampoco alcanzar "la nueva”, sino más bien, por el contrario, "pensar la esencia de la fenomenología más originalmente [que Husserl], para, de esta manera, conducirla de regreso a su propia afiliación con la filosofía occidental"120. En efecto, Husserl parece confirmar con antelación la evaluación de su asistente respecto de su propia originalidad, al decirle a

II3 Cf. Husserl, Edmund, "Carta a Fritz Kaufmann del 20 de abril de 1926”, en: Hua Dok III, III, p. 347.

114 Cf. SZ, p. vy específicamente p. 38.

II5 Cf. Husserl, Edmund, "Carta de Heidegger del 22 de octubre de 1927" y "Carta a Heidegger del 8 de diciembre de 1927", en: Hua Dok III, IV, pp. 144 y I48, respectivamente.

II6 Husserl, Edmund, Recomendación (esbozo), fines de enero de 1928, en: Hua Dok III, VIII, pp. 194-195.

II7 Cf. Husserl, Edmund, “Carta a Heidegger del 2 I de enero de 1928”, en: Hua Dok III, IV, p. I5I. Cf. también Briefwechsel III, p. 457 y Briefwechsel IV, p. 142.

II8 Cf. Husserl, Malvine, "Cartas a Elisabeth Rosenberg" del 22 de junio de 1930 y del 25 de enero de 1933", en: Hua Dok III, IX, pp. 378 y 4I6, respectivamente.

II9 Heidegger, Martin, "Was ist das Sein selbst?" (12 de setiembre de 1946), en: GA 16, p. 423, énfasis añadido.

120 GA I2, p. 91. 
Natorp ya en 1922 que "la manera de ver y de trabajar fenomenológicamente [de Heidegger], así como el campo de sus mismos intereses -nada de esto ha sido meramente tomado de mí, sino más bien que él es capaz erigirse por sí mismo en su propia originalidad" ${ }^{21}$. Así, Ser y tiempo de Heidegger no es solamente el punto de inicio de un cisma, sino también el punto final de un cisma. Ideas I de Husserl es el terminus a quo del "gran cisma fenomenológico" en general, pero Ser y tiempo de Heidegger es el terminus ad quem del mismo cisma, tal como resultó finalmente entre estos dos pensadores en particular. El que Ser y tiempo de Heidegger sea también el terminus a quo del "cisma fenomenológico-existencial”, comúnmente es tomado como algo dado, aunque ello no pueda tomarse sin mayor discusión, puesto que Heidegger tampoco quería tener que ver con la filosofía existencial y el existencialismo.

Finalmente, el mapa del camino de su pensamiento, que Heidegger expone en "Mi camino hacia la fenomenología", es corroborado por lo que él dice sobre su relación con Husserl y posterior ruptura en sus Cuadernos negros ${ }^{122}$, póstumamente publicados. En declaraciones desde 1940, lo que Heidegger dice acerca de la aproximación filosófica de Husserl es mucho más crítico que cualquier cosa que dice sobre ella en "Mi camino hacia la fenomenología”. Dice, por ejemplo, que Husserl, aferrándose al "principio [de] 'a las cosas mismas”" a costa del "pensar las cosas mismas", "no sabe nada de e incluso resiste" la idea del "giro del pensamiento a la 'A $\lambda$ ń $\theta \varepsilon ı \alpha$ como característica esencial del Ser en sí mismo en el sentido de [la] presencia" ${ }^{23}$. A este respecto, Heidegger yuxtapone el "Epílogo a Ideas" y "Filosofía como ciencia rigurosa" de Husserl, de un lado, y las Investigaciones lógicas, del otro' ${ }^{124}$. De acuerdo a Heidegger, el trabajo temprano fue un comienzo en la dirección correcta de "pensar la experiencia de la 'A $\lambda$ ń$\theta \varepsilon 1 \alpha$ más allá de la experiencia del olvido del Ser”, pero no puede decirse lo mismo de las obras posteriores ${ }^{125}$. Para Heidegger, las Investigaciones lógicas, las cuales se arriesgaron a dar "el paso hacia el ver" en oposición a la "argumentación vacía" y la "aserción histórica", aseguraron

I2I Husserl, Edmund, "Carta a Paul Natorp del I de febrero de 1922”, en: Hua Dok III, V, p. 150. Husserl añade que, "como maestro", Heidegger tiene "un efecto propio próximo al mío, y probablemente uno tan fuerte como el mío".

122 Cf. los así llamados "Schwarze Hefte" de Heidegger, en: GA 94-97 ss.

123 GA 97, p. 442. Cf. las observaciones sobre Husserl en GA 97 de fechas 1942-1948.

124 Cf. ibid., p. 442.

$260 \quad 125$ Cf. ibid. 
el "lugar en la historia" de Husserl, mientras que es "precisamente esto" lo que "sus meros defensores y propagandistas no quieren reconocer"126. Para Heidegger, también, "la falsa admiración" por "la filosofía tardía" de Husserl lo hace ver, "en contra de su voluntad", "ridículo" y como "un inepto" 127. No hay duda de que aquí Heidegger está contando al primer volumen de las Ideas relativas a una fenomenología pura y una filosofía fenomenológica, al "Epílogo" y al artículo de la revista Logos ("Filosofía como ciencia rigurosa") conjuntamente, como parte de la "filosofía tardía"128 de Husserl. Bajo estas circunstancias, Heidegger sostiene que él ha "pasado" a Husserl "de largo", agregando que esto fue "una dolorosa necesidad"|29. Aun cuando indica que sus ensayos desde Ser y tiempo son "el testimonio más valioso" de lo que le debe a Husserl, Heidegger enfatiza que "aprendió de él y testificó de ello a su modo, por el hecho de que [él] no permaneció siendo su seguidor, cosa que tampoco nunca lo fue"|30. Para concluir, Heidegger sugiere que uno podría haber reconocido su papel en lo que ahora se considera como el "gran cisma fenomenológico" si uno hubiera consultado sus "conferencias" como "testimonios" de su pensamiento ${ }^{131}$. Todo esto corrobora adicionalmente la observación de Heidegger en "Mi camino hacia la fenomenología" sobre el papel de sus primeras conferencias en Friburgo y Marburgo en lo relativo al desarrollo de su pensamiento que condujera a Ser y tiempo ${ }^{132}$.

\section{$\S 5$. Conclusión: la relación cercana entre los dos cismas}

Como resultado de las investigaciones precedentes de las fuentes, es evidente que, mientras que hay una proyección innegable del "gran cisma fenomenológico" entre Husserl y Pfänder, Scheler, Reinach, Stein e Ingarden, entre 1913 y 1926, en el "cisma fenomenológico-existencial" entre Husserl y Heidegger, entre 1927 y 1933, hay también una superposición abrumadora entre el primer cisma entre Husserl y Heidegger, entre 1917 y 1927, y el segundo cisma

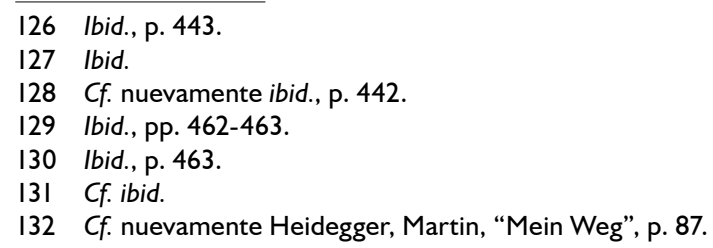


entre Husserl y los otros fenomenólogos, entre 1913 y 1926. Al final, uno ciertamente sigue preguntándose si Husserl y Heidegger estuvieron alguna vez genuinamente próximos filosóficamente hablando, para comenzar. De cualquier manera, el "gran cisma fenomenológico" -entre Husserl y aquellos fenomenólogos que, como Pfänder, Scheler, Reinach, Stein e Ingarden, no siguieron su camino al idealismo transcendental (1905-1913 ss.)- precedió y preparó el "cisma fenomenológico-existencial" entre Husserl y Heidegger (1927-1933 ss.), y solamente es posible comprender esta última ruptura en el horizonte de la primera. En el caso de la relación entre Husserl y Heidegger, y de la relación entre el "gran cisma fenomenológico" y el "cisma fenomenológico-existencial”, sin embargo, es evidente que ambas series de eventos o procesos son distintos pero inseparables, y que se desempeñaron de tal manera que la ruptura fenomenológica temprana de Heidegger respecto de Husserl anticipó su posterior ruptura fenomenológico-existencial respecto de él, así como también que esta última recapituló a la anterior. Al lado de Husserl, Heidegger es el eje de ambos cismas, puesto que participó activamente en cada uno.

Es posible seguir su sugerenciay completar este cuadro con una juiciosa selección de los contenidos de las lecciones de Heidegger en Friburgo de 1919 a 1923 y en Marburgo de 1923 a $1928^{133}$. Por ejemplo, en el semestre de primavera de 1919, Heidegger alega que una filosofía que está comprometida "con el dominio general de lo teorético" o incluso con "la primacía de lo teorético", como lo está [justamente] la fenomenología trascendental de Husserl, puede alcanzar "las cosas mismas" como "objetos", pero que esta aproximación no puede capturar la experiencia humana de la vida histórica en un mundo circundante ("es weltet") 134. En el semestre de invierno de 1919/1920, Heidegger pregunta, por cierto en lo que respecta a la definición de Husserl de la "filosofía como ciencia rigurosa" en su artículo de la revista Logos de 1911, "qué queda entonces" de "los ideales de la fenomenología como investigación rigurosa"|35. En el semestre de invierno de 1920/1921, Heidegger propone que la fenomenología de Husserl gire hacia "lo histórico"|36. En el semestre de invierno de 1921/1922

133 Cf. ibid. y GA 97, p. 463.

134 Cf. GA 56/57, pp. 73, 75, 87, II7.

135 GA 58, p. 141.

262136 GA 60, p. 31 ss. 
y el semestre de verano de 1922, Heidegger, orientándose cada vez más hacia Aristóteles y cada vez menos hacia Husserl, desarrolla su "fenomenología de la vida" y su concepto de filosofía como el análisis de la "vida fáctica" o "la facticidad de la vida fáctica" ${ }^{37}$. En el semestre de verano de 1923, Heidegger aplica el concepto de la facticidad a la existencia (Dasein), insta a sus oyentes a llevar a cabo el giro hermenéutico en fenomenología y sugiere que Husserl, a pesar de sus "contribuciones decisivas", no lo hizo ${ }^{138}$. En el semestre de invierno de 1923/1924, Heidegger critica a Husserl por su cartesianismo, esto es, por estar "obsesionado con la conciencia y ciego para la vida", de modo que no consigue plantear la pregunta por el Sein de la Bewusstsein ${ }^{139}$. En el semestre deverano de 1925, Heidegger culpa a Husserl por haber absolutizado los ideales científicos de las ciencias matemáticas y naturales en detrimento de las Geisteswissenschaften, así como por haber favorecido lo teorético en detrimento de lo existencial ${ }^{140}$. En el semestre de invierno de 1925/1926, Heidegger cuestiona el fundamento de la crítica de Husserl al psicologismo, a saber, la distinción rigurosa entre el acto real de juzgar y el contenido ideal del juicio, la cual, de acuerdo a Heidegger, define la verdad como un carácter ideal de ser pero no aclara su Ser realmente existente ${ }^{|4|}$. Sería fácil continuar esta lista, pero el punto es obvio. Hay también aquí una fuerte evidencia de que la ruptura temprana de Heidegger respecto de Husserl fue el resultado de su insatisfacción y rechazo de la fenomenología tal como fue redefinida por Ideas I, y que la ruptura temprana fue un proceso en progreso varios años antes de la aparición de Ser y tiempo.

Los puntos de encuentro entre la crítica filosófica de Heidegger a Husserl luego de su transformación de la fenomenología descriptiva en fenomenología trascendental, de un lado, y las críticas filosóficas latentes y patentes a Husserl por parte de Pfänder, Scheler, Reinach, Stein e Ingarden, de otro, son también evidentes. Desde Los fundamentos a priori de la ley civil (1913) de Reinach ${ }^{142}$, pasando por El formalismo en la ética y la ética material de los valores (1913-1916)

\footnotetext{
137 GA 6I, p. 79 ss.

138 Cf. GA 63, pp. 16, 77.

139 Cf. GA 17, pp. 256, 267, 274-275.

140 Cf. GA 20, § 4, específicamente la concluyente subsección sobre Husserl.

I4I Cf. GA 2I, pp. 54, 58. Cf. SZ, § 44.

142 Cf. Reinach, Adolf, "Die apriorischen Grundlagen des Bürgerlichen Rechts (1913)", en: Adolf Reinach: Sämtliche Werke, vol. I, pp. 14|-278.
} 
de Scheler ${ }^{143}$, La obra de arte literaria: una investigación desde la región fronteriza de la ontología, la lógica y la erudición literaria (193I) de Ingarden ${ }^{144}$ y el Ser finito y eterno: intento de ascenso al sentido del ser (193I-1936) de Stein ${ }^{145}$ a El alma del ser humano (1933) de Pfänder ${ }^{146}$, las obras distintivas de estos fenomenólogos muestran que ellos estaban ya empujando contra los límites de la fenomenología descriptiva tal como Husserl la había redefinido en su movimiento hacia el idealismo trascendental. Si uno toma también en consideración Sobre el problema de la empatía de Stein (1917) $)^{147}$ y El puesto del hombre en el cosmos de Scheler $(1928)^{148}$, además de permitir una lectura cuestionable pero plausible de Ser y tiempo de Heidegger como una filosofía de la existencia acompañada de una antropología filosófica (sin perjuicio de la crítica de Stein a la obra ${ }^{149}$, entonces se aclara por qué Husserl malinterpretó la situación en evolución y percibió a la fenomenología como [estando] casi bajo ataque de parte de la antropología y la filosofía de la existencia. Esta interpretación de eventos es confirmada por la distinción posterior [que realiza] Husserl entre los tópicos que la filosofía debe tratar y los tópicos que la fenomenología transcendental, con su método de la reducción trascendental, no puede manejar, esto es, "los problemas límite o fronterizos de la fenomenología"150. Evidentemente Husserl cree que los Grenzprobleme der Phänomenologie incluyen la pregunta por el sentido de la vida humana ${ }^{151}$. No es, por supuesto, como si Husserl no planteara "las preguntas sobre el sentido o el sinsentido de toda esta existencia humana"152. Es el caso, no obstante, que Heidegger no pensó que estas preguntas existenciales pudieran abordarse desde el interior de la actitud

143 Cf. Scheler, Max, Der Formalismus in der Ethik und die materiale Wertethik: Neuer Versuch der Grundlegung eines ethischen Personalismus (1913-1916) (GW II).

144 Cf. Ingarden, Roman, Das literarische Kunstwerk: Eine Untersuchung aus dem Grenzgebiet der Ontologie, Logik und Literaturwissenschaft (193I).

145 Cf. Stein, Edith, "Endliches und ewiges Sein: Versuch eines Aufstiegs zum Sinn des Seins (193I-1936)", en: GA II/I2, pp. 3-44I.

146 Cf. Pfänder, Alexander, Die Seele des Menschen (1933).

147 Cf. Stein, Edith, "Zum Problem der Einfühlung (1917)", en: GA 5, pp. I-I36.

148 Cf. Scheler, Max, GW IX, pp. 7-72.

149 Cf. Stein, Edith, “Martin Heideggers Existenzphilosophie(I937)”, en: GA II/I2, pp. 445-499.

I50 Husserl mismo no usa el término "Grenzprobleme"; sin embargo, el concepto está presente. Cf. Hua XXXIX, pp. 875-876, así como la "Introducción de los editores" en Hua XLII, pp. xix-cxv, específicamente p. xix, nota $\mathrm{I}$.

I5I Hua XLII incluso contiene un texto (el No. 18, probablemente de 1934) en el cual Husserl menciona "Existenzphilosophie", pero sin decir nada malo sobre ello (cf. Hua XLII, pp. 228-235). 
trascendental de las /deas de Husserl ${ }^{153}$. Sin embargo, estas cuestiones podrían abordarse propiamente en otros artículos.

Traducción de Emily Noejovich y Rosemary Rizo-Patrón

Copyright 2015 by George Heffernan, george.heffernan@merrimack.edu

I53 Cf. Husserl, Edmund, "Carta de Heidegger del 22 de octubre de 1927" y "Carta a Heidegger del 8 de diciembre de 1927”, en: Hua Dok III, IV, pp. I44-I48 y I49, respectivamente. 


\section{Bibliografía}

Arendt, Hannah, “What Is Existenz Philosophy?”, en: Partisan Review, XIII (1946), pp. 34-56.

— “Was ist Existenz-Philosophie?”, en: Sternberger, Dolf (ed.), Schriften der Wandlung

3: Hannah Arendt - Sechs Essays, Heidelberg: Lambert Schneider, 1948, pp. 48-80.

— “What Is Existential Philosophy?", traducción de Robert y Rita Kimber, en: Kohn, Jerome (ed.), Hannah Arendt. Essays in Understanding 1930-1954, Nueva York: Harcourt Brace \& Company, 1994, pp. 163-187.

Arendt, Hannah y Martin Heidegger, Briefe 1925 bis 1975 und andere Zeugnisse, edición de Ursula Ludz, 3ra. edición, Frankfurt am Main: Vittorio Klostermann, 2002.

Arendt, Hannah y Karl Jaspers, Briefwechsel 1926-1969, edición de Lotte Köhler y Hans Saner, Munich: R. Piper Verlag, 1985.

Barua, Archana, "Husserl, Heidegger, and the Transcendental Dimension of Phenomenology”, en: Indo-Pacific Journal of Phenomenology, VII (2007), pp. I-I0.

Beck, Maximilian, “The Last Phase of Husserl's Phenomenology: An Exposition and a Criticism”, en: Philosophy and Phenomenological Research, I (194I), pp. 479-49I.

— “In Reply to Cairns' Critical Remarks”, en: Philosophy and Phenomenological Research, I (I94I), p. 498.

— “Existentialism”, en: Philosophy and Phenomenological Research, V(1944), pp. I26-I37.

— "Reason and Existence", en: Journal of Philosophy, XLIV (I947), pp. 375-380.

Bernet, Rudolf, Alfred Denker y Holger Zaborowski (eds.), Heidegger und Husserl (Heidegger-Jahrbuch 6), Freiburg/Munich: Karl Alber, 2012.

Biemel, Walter, "Husserls Encyclopaedia-Britannica-Artikel und Heideggers Anmerkungen dazu”, en: Tijdschrift voor Philosophie, XII (1950), pp. 246-280.

Boyce Gibson, William R., "From Husserl to Heidegger: Excerpts from a 1928 Freiburg Diary, edited by Herbert Spiegelberg”, en: Journal of the British Society for Phenomenology, II (197I), pp. 58-83.

Braig, Carl, Vom Sein: Abriß der Ontologie, Freiburg im Breisgau: Herder, 1896.

Breeur, Roland (ed.), "Randbemerkungen Husserls zu Heideggers Sein und Zeit und Kant und das Problem der Metaphysik”, en: Husserl Studies, XI (1994), pp. 3-63.

Brentano, Franz, Von der mannigfachen Bedeutung des Seienden nach Aristoteles, Freiburg im Breisgau: Herder, 1862.

Cairns, Dorion, “Concerning Beck's 'The Last Phase of Husserl's Phenomenology”, en: Philosophy and Phenomenological Research, I (194I), pp. 492-498. 
Celms, Theodor, Der phänomenologische Idealismus Husserls. Riga: Latvijas Universitates raksti / Acta Universitatis Latviensis, 1928.

Courtine, Jean-François (ed.), Heidegger 1919-1929: de l'herméneutique de la facticité à la métaphysique du Dasein, París: J. Vrin, 1996.

Cristin, Renato (ed.), Edmund Husserl - Martin Heidegger - Phänomenologie (1927), Berlín: Duncker \& Humblot, 1999.

Crowell, Steven, “Husserl, Heidegger, and Transcendental Philosophy: Another Look at the Encyclopaedia Britannica Article”, en: Philosophy and Phenomenological Research, L (1990), pp. 50I-5I8.

- Husserl, Heidegger, and the Space of Meaning: Paths Toward Transcendental Phenomenology, Evanston: Northwestern University Press, 2001.

— “Does the Husserl/Heidegger Feud Rest on a Mistake?", en: Husserl Studies, XVIII (2002), pp. 123-140.

Dahlstrom, Daniel, “Heidegger's Transcendentalism”, en: Research in Phenomenology, XXXV (2005), pp. 29-54.

Denker, Alfred, Unterwegs in “Sein und Zeit”: Einführung in Leben und Denken von Martin Heidegger, Stuttgart: Klett-Cotta, 2011.

“Martin Heidegger's Being and Time: A Carefully Planned Accident?”, en: Mark

Wrathall (ed.), The Cambridge Companion to Heidegger's “Being and Time”, Cambridge: Cambridge University Press, 2013, pp. 54-83.

Hachmeister, Lutz, Heideggers Testament: Der Philosoph, der "Spiegel" und die SS, 2da. edición, Berlín: Propyläen / Ullstein, 2014.

Heidegger, Martin, Martin Heidegger: Gesamtausgabe, Frankfurt am Main: Vittorio Klostermann, 102 vols., 1977 ss. (abreviado como GA).

GA I (1978), Frühe Schriften (1912-1916), edición de F.-W. Von Herrmann, pp. I-I29 ("Die Lehre vom Urteil im Psychologismus: Ein kritisch-positiver Beitrag zur Logik [1913/1914]”), pp. I31-353 (“Die Kategorien- und Bedeutungslehre des Duns Scotus [1915/1916]")

GA 2 [1977]), Sein und Zeit (1927), edición de F.-W. Von Herrmann (también en:..14ta. edición, Halle / Tubinga: Max Niemeyer, 1977).

GA 3 (2da. edición, 2010), Kant und das Problem der Metaphysik (1929), edición de F.-W. Von Herrmann.

GA9 (3ra. edición, 2004), Wegmarken (1919-196I), edición de F.-W. Von Herrmann, pp. I-44 (“Anmerkungen zu Karl Jaspers Psychologie der Weltanschauungen" [1919-192I]), pp. 103-122 (“Was ist Metaphysik?” [1929]), pp. 313-364 (“Über den Humanismus” [1945]; también en Frankfurt am Main: Vittorio Klostermann, 2010, IIra. edición). GA 12 (1985), Unterwegs zur Sprache (1950-1959), edición de F.-W. Von Herrmann. GA 14 (2007), Zur Sache des Denkens, edición de F.-W. Von Herrmann, pp. 8I-90 (“Mein Weg in die Phänomenologie [1963/1969]”; también en Tubinga: Max Niemeyer Verlag, 1988, 3ra. edición). 
GA 16 (2000), Reden und andere Zeugnisse eines Lebensweges 1910-1976, edición de H. Heidegger, pp. 423-425, (“Was ist das Sein selbst?” [12 de setiembre de 1946]). GA 17 (2da. edición, 2006), Einführung in die phänomenologische Forschung (1923/1924), edición de F.-W. Von Herrmann.

GA I8(2002), Grundbegriffe der aristotelischen Philosophie (1924), edición de M. Michalski. GA 20 (1979), Prolegomena zur Geschichte des Zeitbegriffs (1925), edición de P. Jaeger. GA 2I (1976), Logik. Die Frage nach der Wahrheit (1925-1926), edición de Walter Biemel. GA 24 (3ra. edición, 1997), Die Grundprobleme der Phänomenologie (1927), edición de F.-W. Von Herrmann.

GA 27 (1996), Einleitung in die Philosophie (1928/1929), edición de O. Saame e I. Saame-Speidel.

GA 32 (1980), Hegels Phänomenologie des Geistes (1930/193I), edición de I. Görland.

GA 49 (199I), Die Metaphysik des deutschen Idealismus. Zur erneuten Auslegung von Schelling: Philosophische Untersuchungen über das Wesen der menschlichen Freiheit und die damit zusammenhängenden Gegenstände (194I), edición de G. Seubold.

GA 56/57 (1987), Zur Bestimmung der Philosophie (1919), edición de B. Heimbüchel.

GA 58 (1992), Grundprobleme der Phänomenologie (1919/1920), edición de H. H. Gander. GA 60 (1995), Phänomenologie des religiosen Lebens (1920-192I), edición de M. Jung y T. Regehly.

GA $6 I$ (2da. edición, 1994), Phänomenologische Interpretationen zu Aristoteles: Einführung in die phänomenologische Forschung (1921/1922), edición de W. Bröcker y K. Bröcker-Oltmanns.

GA 62 (2005), Phänomenologische Interpretation ausgewählter Abhandlungen des Aristoteles zu Ontologie und Logik (1922), edición de G. Neumann.

GA63(1988), Ontologie. Hermeneutik der Faktizität (1923), edición de K. Bröcker-Oltmanns. GA 94 (20I4), Überlegungen II-VI (Schwarze Hefte I93I-1938), edición de P. Trawny. GA 95 (2014), Überlegungen VII-XI (Schwarze Hefte 1938/39), edición de P. Trawny. GA 96 (20I5), Überlegungen XII-XV(Schwarze Hefte I939-194I), edición de P. Trawny. GA 97 (20I5), Anmerkungen I-V (Schwarze Hefte 1942-1948), edición de P. Trawny.

— Being and Time, traducción al inglés de John Macquarrie y Edward Robinson, Nueva York: Harper and Row Publishers, 1962.

— "My Way into Phenomenology”, en: On Time and Being, traducción al inglés de Joan Stambaugh, Nueva York: Harper and Publishers, 1972, pp. 74-82.

— "Nur noch ein Gott kann uns retten" (23 de setiembre de 1966), en: Der Spiegel, XXIII (3I de mayo de 1976), pp. 193-219.

— “'Only a God Can Save Us': The Spiegel Interview (1966)”, traducción al inglés de William Richardson, en: Sheehan, Thomas (ed.), Heidegger: The Man and the Thinker, Piscataway: Transaction Publishers, 198I, pp. 45-67.

— "Brief Martin Heideggers an Elisabeth Husserl" (24 de abril de 1919), en: Aut-aut, 223-224 (1988), pp. 6-II.

— “Drei Briefe Martin Heideggers an Löwith”, en: Papenfuss, Dietrich y Otto Pöggeler (eds.), Zur philosophischen Aktualität Heideggers 2: Im Gespräch der Zeit, Frankfurt am Main: Vittorio Klostermann, 1990, pp. 27-39.

— "Mein liebes Seelchen!" Briefe Martin Heideggers an seine Frau Elfride (1915-1970), edición de Gertrud Heidegger, Munich: Deutsche Verlags-Anstalt, 2005. 
Heidegger, Martin y Elisabeth Blochmann, Briefwechsel 1918-1969, edición de Joachim Storck, Marbach am Neckar: Deutsche Schillergesellschaft, 1989.

Heidegger, Martin y Karl Jaspers, Briefwechsel 1920-1963, edición de Walter Biemel y Hans Saner, Frankfurt am Main: Vittorio Klostermann, 1990.

Heinemann, Fritz, Neue Wege der Philosophie: Geist-Leben-Existenz. Eine Einführung in die Philosophie der Gegenwart, Leipzig: Queller und Meyer, 1929.

— "Was ist lebendig und was ist tot in der Existenzphilosophie?", en: Zeitschrift für philosophische Forschung, V (1950), pp. 3-24.

— Existenzphilosophie -lebendig oder tot?, Stuttgart: Kohlhammer, 1954.

Husserl, Edmund, Edmund Husserl: Gesammelte Werke. Husserliana, La Haya/Dordrecht / Nueva York: Martinus Nijhoff / Kluwer Academic Publishers / Springer, 42 vols., 1950 ss. (abreviado como Hua, seguido del volume en números romanos).

Hua II (1973), Die Idee der Phänomenologie: Fünf Vorlesungen (1907), edición de Walter Biemel, 2da. edición.

Hua III/I-2 (1976), Ideen zu einer reinen Phänomenologie und phänomenologischen Philosophie. Erstes Buch: Allgemeine Einführung in die reine Phänomenologie (1913), edición de Karl Schuhmann.

Hua V (2da. edición, 197I), Ideen zur einer reinen Phänomenologie und phänomenologischen Philosophie. Drittes Buch: Die Phänomenologie und die Fundamente der Wissenschaften, edición de Marly Biemel, pp. 138-I62 ("Nachwort zu meinen Ideen zu einer reinen Phänomenologie und phänomenologischen Philosophie” [1930]).

Hua VI (1976), Die Krisis der europäischen Wissenschaften und die transzendentale Phänomenologie. Eine Einleitung in die phänomenologische Philosophie, edición de Walter Biemel. Hua IX(2da. edición, 1968), Phänomenologische Psychologie. Vorlesungen Sommersemester (1925), edición de Walter Biemel, pp. 237-30I ("Der Encyclopaedia Britannica Artikel” [1927-1928]), pp. 302-349 (“Amsterdamer Vorträge: Phänomenologische Psychologie” [1928]).

Hua X(1966), Zur Phänomenologie des inneren Zeitbewusstesens (1893-1917), edición de RudolfBoehm, pp. 236-268 (“Seefelder Manuskripte über Individuation” [1905-1907]). Hua XVII (1974), Formale and transzendentale Logik. Versuch einer Kritik der logischen Vernunft (1929), edición de Paul Janssen.

Hua XVIII (1975), Logische Untersuchungen. Erster Band: Prolegomena zur reinen Logik (1900/1913), edición de Elmar Holenstein.

Hua XIX/I-2 (1984), Logische Untersuchungen: Untersuchungen zur Phänomenologie und Theorie der Erkenntnis (1901/1913/192I), edición de Ursula Panzer.

Hua XX/I (2002), Logische Untersuchungen. Ergänzungsband. Erster Teil (Sommer 1913), edición de Ullrich Melle, pp. 272-329 (“Entwurf einer 'Vorrede' zu den Logischen Untersuchungen” [1913], también en: Tijdschrift voor Filosofie, I (1939), pp. 106-133, 319-339). Hua XX/2 (2005), Logische Untersuchungen. Ergänzungsband. Zweiter Teil (I893/941921), edición de Ullrich Melle.

Hua XXV (1987), Aufsätze und Vorträge (1911-192I), Mit ergänzenden Texten, edición de Thomas Nenon y Hans-Rainer Sepp, pp. 3-62 ("Philosophie als strenge Wissenschaft" [I9II]). 
Hua XXVII (1989), Aufsätze und Vorträge (1922-1937), edición de Thomas Nenon y Hans-Rainer Sepp, pp. 164-18I ("Phänomenologie und Anthropologie” [193I]).

Hua XXIX (1992), Die Krisis der europäischen Wissenschaften und die transzendentale Phänomenologie. Ergänzungsband. Texte aus dem Nachlass 1934-1937, edición de Reinhold N. Smid.

Hua XXXIX, Die Lebenswelt. Auslegungen der vorgegebenen Welt und ihrer Konstitution. Texte aus dem Nachlass (1916-1937), edición de Rochus Sowa.

Hua XLII (20I4), Grenzprobleme der Phänomenologie. Analysen des Unbewusstseins und der Instinkte. Metaphysik. Späte Ethik (Texte aus dem Nachlass 1908-1937), edición de Rochus Sowa y Thomas Vongehr.

- Husserliana Dokumente, edición de Karl Schuhmann et al., The Hague/Dordrecht: Martinus Nijhoff/Kluwer Academic Publishers/Springer, 1977-1999.

Hua Dok I (1977), Husserl-Chronik: Denk- und Lebensweg Edmund Husserls.

Hua Dok III (1994), Briefwechsel, I0 sub-vols. (abreviado como Hua Dok, seguido del número del sub-volumen).

- Husserliana Materialien, edición de Elisabeth Schuhmann et al., Dordrecht/New York: Kluwer Academic Publishers/Springer, 9 vols, 200I-20I2 (abreviado como Hua Mat, seguido del número del volumen)

- Psychological and Transcendental Phenomenology and the Confrontation with Heidegger (1927-193I), edición y traducción al inglés de Thomas Sheehan y Richard Palmer, Dordrecht: Kluwer Academic Publishers, 1997.

Ingarden, Roman, “Bemerkungen zum Problem 'Idealismus-Realismus”, en: Festschrift E. Husserl zum 70. Geburtstag gewidmet. Ergänzungsband zum Jahrbuch für Philosophie und phänomenologische Forschung, X (1929), pp. 159-190.

- Das literarische Kunstwerk: Eine Untersuchung aus dem Grenzgebiet der Ontologie, Logik und Literaturwissenschaft (193I), 2da. edición, Halle: Max Niemeyer, 1960.

_ “Über den transzendentalen Idealismus bei E. Husserl”, en: Van Breda, Herman Leo y Jacques Taminiaux (eds.), Husserl et la Pensée Moderne - Husserl und das Denken der Neuzeit, La Haya: Martinus Nijhoff, 1959, pp. 190-204.

- On the Motives which Led Husserl to Transcendental Idealism, traducción al inglés de Arnor Hannibalsson, La Haya: Martinus Nijhoff, 1975.

Jamme, Christoph y Otto Pöggeler (eds.), Phänomenologie im Widerstreit: Zum 50. Todestag Edmund Husserls, Frankfurt am Main: Suhrkamp, 1989.

Jaspers, Karl, Psychologie der Weltanschauungen (1919), 6ta. edición, Berlín:Springer, 1971. - Existenzphilosophie: Drei Vorlesungen gehalten am Freien Deutschen Hochstift in Frankfurt a.M. September 1937, 2da. edición, Berlín: Walter de Gruyter, 1956.

Kisiel, Theodore, The Genesis of Heidegger's “Being and Time”, Berkeley: University of California Press, 1993.

- Heidegger's Way of Thought: Critical and Interpretative Signposts, edición de Alfred Denker y Marion Heinz, Nueva York: Continuum, 2002.

Landgrebe, Ludwig, “Husserls Phänomenologie und die Motive zu ihrer Umbildung”, Revue Internationale de Philosophie, I (1939), pp. 277-316. 
Lask, Emil, Die Logik der Philosophie und die Kategorienlehre: Eine Studie über den Herrschaftsbereich der logischen Form, Tubinga: J.C.B. Mohr, 1911.

— Die Lehre vom Urteil, Tubinga: J.C.B. Mohr, 1912.

Löwith, Karl, Mein Leben in Deutschland vor und nach 1933: Ein Bericht (1940), edición de Frank-Rutger Hausmann, 2da. edición, Stuttgart: J.B. Metzler, 2007.

Luft, Sebastian, “Muss Philosophie als transzendentaler Idealismus auftreten? Zur 'wahren Gestalt' der Philosophie bei Husserl und seinen Vorgängern im Deutschen Idealismus", en: Luft, Sebastian y Faustino Fabbianelli (eds.), Husserl und die klassische deutsche Philosophie, Dordrecht: Springer, 2014, pp. 29-47.

Misch, Georg, Lebensphilosophie und Phänomenologie: Eine Auseinandersetzung der Diltheyschen Richtung mit Heidegger und Husserl, Stuttgart: B.G. Teubner, 1967.

Ott, Hugo, Martin Heidegger: Unterwegs zu seiner Biographie, Frankfurt am Main: Campus Verlag, 1988.

Petzet, Heinrich Wiegand, Auf einen Stern zugehen: Begegnungen und Gespräche mit Martin Heidegger, 1929 bis 1976, Frankfurt am Main: Societäts Verlag, 1983.

Pfänder, Alexander, Phänomenologie des Wollens: Eine psychologische Analyse, Leipzig: J.A. Barth, 1900.

— Einführung in die Psychologie, Leipzig: J.A. Barth, 1904.

— "Zur Psychologie der Gesinnungen”, en: Jahrbuch für Philosophie und phänomenologische Forschung, I (1913), pp. 325-404 y III (1916), pp. I-I25.

— Logik, en: Jahrbuch für Philosophie und phänomenologische Forschung, IV (192I), pp. 139-494e.

— Die Seele des Menschen, Halle: Max Niemeyer, 1933.

Pöggeler, Otto, Der Denkweg Martin Heideggers, 2da. edición, Pfullingen: Günther Neske, 1983.

Reinach, Adolf, Sämtliche Werke, edición de Karl Schuhmann y Barry Smith, Munich: Philosophia Verlag, 1989, 2 vols.

— Vol. I (1989), “Die apriorischen Grundlagen des Bürgerlichen Rechts (1913)”, pp. 14|-278;y “Vortrag über Phänomenologie (1914/I92I)”, pp. 531-550 (este texto también en: Was ist Phänomenologie?, Munich: Kösel, 195I).

Rickert, Heinrich, Der Gegenstand der Erkenntnis: Einführung in die Transzendentalphilosophie (1892), 3ra. edición, Freiburg im Breisgau: Herder, 1915.

Safranski, Rüdiger, Ein Meister aus Deutschland: Heidegger und seine Zeit, 2da. edición, Munich: Carl Hanser Verlag, 1995.

Sartre, Jean-Paul, “L'existentialisme est un humanisme” (1946), edición de Arlette Elkaïm-Sartre, París: Gallimard, 1996. 
Scheler, Max, Gesammelte Werke, 16 vols., I, Berna: Francke, 1954-1998, se citará como GW, seguido del número del volumen.

— Vol. I (197I), "Die transzendentale und die psychologische Methode: Eine grundsätzliche Erörterung zur philosophischen Methode (1900/1922)”, pp. 197-336.

— Vol. II (1954), Der Formalismus in der Ethik und die materiale Wertethik: Neuer Versuch der Grundlegung eines ethischen Personalismus (1913-1916), 4ta. edición.

— Vol. III (1955), "Versuche einer Philosophie des Lebens: Nietzsche - Dilthey Bergson” (1913), 4ta. edición, pp. 3II-340.

— Vol. V (1954), Vom Ewigen im Menschen (192I).

— Vol. VII (1973), "Wesen und Formen der Sympathie (1913)”, y “Zur Phänomenologie und Theorie der Sympathiegefühle und von Liebe und Hass (19/3)”, pp. 7-258.

— Vol. IX (1976), “Die Stellung des Menschen im Kosmos (1928)”, pp. 7-72, y “Philosophische Weltanschauung (1929)”, pp. 73-182.

Schuhmann, Karl, “Zu Heideggers Spiegel-Gespräch über Husserl”, en: Zeitschrift für philosophische Forschung, XXXII (1978), pp. 59I-6I2.

Schuhmann, Karl y Barry Smith, “Against Idealism: Johannes Daubert vs. Husserl's Ideas I”, en: Review of Metaphysics, XXXIX (1985), pp. 763-793.

Sheehan, Thomas (ed.), Heidegger: The Man and the Thinker, Piscataway: Transaction Publishers, 1981.

Sheehan, Thomas y Richard Palmer (eds.), Edmund Husserl: Psychological and Transcendental Phenomenology and the Confrontation with Heidegger (1927-193I), Dordrecht: Kluwer Academic Publishers, 1997.

Spiegelberg, Herbert, The Phenomenological Movement: A Historical Introduction, 3ra. edición, La Haya: Martinus Nijhoff, 1982.

Stein, Edith, Gesamtausgabe, 27 vols, Freiburg im Breisgau: Herder, diversos editores. Se citará con la sigla GA seguida del número del volumen.

— GA 9 (2014), Beiträge zu Phänomenologie und Ontologie, pp. 85-90 (“Was ist Phänomenologie? [1924]”); pp. I43-I58 (“Die weltanschauliche Bedeutung der Phänomenologie [1930/I93I]"); pp. 159-16I (“Husserls transzendentale Phänomenologie [193I])". — GA II/I2 (2013, 2da. edición), Endliches und ewiges Sein, Versuch eines Aufstiegs zum Sinn des Seins, pp. 3-44I ("Endliches und ewiges Sein: Versuch eines Aufstiegs zum Sinn des Seins [1931-1936])”), y pp. 445-499 (“Martin Heideggers Existenzphilosophie" [1937])".

— GA 14 (2010, 2da edición), Der Aufbau der menschlichen Person: Vorlesung zur philosophischen Anthropologie (1932), pp. I-172.

— GA I5 (2005), Was ist der Mensch? Theologische Anthropologie (1933).

Thomä, Dieter (ed.), Heidegger Handbuch: Leben-Werk-Wirkung, 2da. edición, Stuttgart: J.B. Metzler, 2013.

Vetter, Helmuth, Grundriss Heidegger: Ein Handbuch zu Leben und Werk, Hamburg: Felix Meiner Verlag, 2014. 